\title{
On permutation complexity of fixed points of some uniform binary morphisms
}

\author{
Alexandr Valyuzhenich $]^{*}$ \\ Department of Mechanics and Mathematics, Novosibirsk State University, Russia
}

received $12^{\text {th }}$ Sep. 2012, revised $3^{\text {rd }}$ June 2014, accepted 27th June 2014.

We study properties of infinite permutations generated by fixed points of some uniform binary morphisms, and find a precise formula for their complexity.

Keywords: infinite permutation, factor complexity, morphism

\section{Introduction}

A D0L word $\omega$ is an infinite word on a finite alphabet $\Sigma$ which is a fixed point of a morphism $\varphi: \Sigma^{*} \longrightarrow$ $\Sigma^{*}$, i.e., $\omega=\lim _{n \rightarrow \infty} \varphi^{n}(a)$ for $a \in \Sigma$. The class of D0L words has been extensively studied and contains famous words such as the cube-free Thue-Morse word and a square-free word on the three-letter alphabet. The subword complexity $C(n)$ of a word $\omega$ is the number of distinct words of length $n$ which occur in $\omega$. This function on infinite words has been studied in numerous papers; see, for instance, the survey Cassaigne and Nicolas (2010). The subword complexity of D0L words is well studied. For instance, in Cassaigne (1997), a general method for calculating the subword complexity of DOL words was developed, and in Frid (1998), the subword complexity of marked uniform D0L words was found. The subword complexity of the Thue-Morse word was found in Brlek (1989), Luca and Varricchio (1989), and later in Avgustinovich (1994).

In this paper we study infinite permutations generated by infinite DOL words. The notion of an infinite permutation was introduced in Fon-Der-Flaass and Frid (2007), where periodic properties and low complexity of permutations were investigated. Similarly to the definition of subword complexity of infinite words, we can introduce the factor complexity of a permutation as the number of its distinct subpermutations of a given length. Another complexity function called maximal pattern complexity of infinite permutations was investigated in Avgustinovich et al. (2011).

The notion of a permutation generated by an infinite non-periodic word was introduced by Makarov (2006). In Makarov (2009) the same author calculated the factor complexity of permutations generated

*Email: graphkiper@mail.ru. The work is supported by grant of RFFI (no. 12-01-00089-a) and (no. 13-01-00463-a). 
by the well-known family of Sturmian words. Widmer (2011) calculated the factor complexity of the permutation generated by the Thue-Morse word.

In this paper we find a more general formula for the factor complexity of permutations generated by fixed points of binary uniform morphisms from a wide class. In particular, since the Thue-Morse word belongs to this class, we obtain an alternative way to compute the factor complexity of the Thue-Morse permutation.

This paper is the extended and modified version of initial conference paper Valyuzhenich (2011). We added proofs. Also we solve recurrent relations from Valyuzhenich (2011) and find the exact formula for the permutation complexity. We calculate the permutation complexity for a wider class of morphisms.

In Section 2 we introduce basic definitions to be used below. In Section 3 we give the main ideas of our proof. In Section 4 , we introduce the class of morphisms $Q_{l}$ for which the main theorem of this paper stated in Section 8 is valid. In Sections $5-7$ we state some auxiliary statements needed to state and prove the main theorem. In Section 9 we give an alternative proof of the formula for the factor complexity of the Thue-Morse permutation.

\section{Basic definitions}

Let $\Sigma$ be a finite alphabet. Everywhere below we will use only the two-letter alphabet $\Sigma=\{0,1\}$.

An infinite word over the alphabet $\Sigma$ is a word of the form $\omega=\omega_{1} \omega_{2} \omega_{3} \ldots$, where $\omega_{i} \in \Sigma$. A (finite) word $u$ is called a subword or factor of a (finite or infinite) word $v$ if $v=s_{1} u s_{2}$ for some words $s_{1}$ and $s_{2}$ which may be empty. The length of a finite word $u$ is denoted by $|u|$. The set of all finite subwords of the word $\omega$ is denoted by $F(\omega)$.

A mapping $h: \Sigma^{*} \longrightarrow \Sigma^{*}$ is called a morphism if $h(x y)=h(x) h(y)$ for any words $x, y \in \Sigma^{*}$. We say that $\omega$ is a fixed point of a morphism $\varphi$ if $\varphi(\omega)=\omega$. Clearly, every morphism is uniquely determined by the images of letters, which are called blocks. A morphism is called l-uniform if its blocks are of the same length $l$.

We say that a morphism $\varphi: \Sigma^{*} \longrightarrow \Sigma^{*}$ is marked if its blocks are of the form $\varphi\left(a_{i}\right)=b_{i} x_{i} c_{i}$, where $x_{i}$ is an arbitrary word, $b_{i}$ and $c_{i}$ are symbols of the alphabet $\Sigma$, and all $b_{i}$ (as well as all $c_{i}$ ) are distinct. In what follows, we will consider only binary $l$-uniform marked morphisms.

We define $\varphi_{i ; j}(v)$ as the word obtained from $\varphi(v)$ by erasing $i$ symbols to the left and $j$ symbols to the right where $0 \leq i<l$ and $0 \leq j<l$. An interpretation of a word $u \in \Sigma^{*}$ under a morphism $\varphi$ is a triple $s=\langle v, i, j\rangle$, where $v=v_{1} \ldots v_{k}$ is a word over the alphabet $\Sigma, i$ and $j$ are nonnegative integers such that $0 \leq i<\left|\varphi\left(v_{1}\right)\right|$ and $0 \leq j<\left|\varphi\left(v_{k}\right)\right|$, and $u=\varphi_{i ; j}(v)$. In addition, if $v$ is a subword of $\omega=\varphi(\omega)$, then $s$ is called an interpretation on $\omega$. The word $v$ is called an ancestor of the word $u$. In what follows we shall consider only interpretations on $\omega$. We say that $\left(u_{1}, u_{2}\right)$ is a synchronization point (see Cassaigne (1994)) of $u \in F(\omega)$ if $u=u_{1} u_{2}$ and $\forall v_{1}, v_{2} \in \Sigma^{*}, \forall s \in F(\omega) \exists s_{1}, s_{2} \in F(\omega)$ such that $\left[v_{1} u v_{2}=\varphi(s) \Rightarrow\left(s=s_{1} s_{2}, v_{1} u_{1}=\varphi\left(s_{1}\right), u_{2} v_{2}=\varphi\left(s_{2}\right)\right)\right]$. A fixed point $\omega=\varphi(\omega)$ of the morphism $\varphi$ is called circular (see Cassaigne (1994)) with synchronization delay $L_{\omega}$, if $L_{\omega}$ is an integer such that any subword $v$ of word $\omega$ of length at least $L_{\omega}$ contains at least one synchronization point.

Remark 1 If $\varphi$ is marked, then circularity of a word $\omega=\varphi(\omega)$ means that any of its subwords $u$ with $|u| \geq L_{\omega}$ admits a unique interpretation on $\omega:$ as we know a synchronization point, we can reconstruct every block of this interpretation from its first (or last) symbol. Moreover for marked morphisms, this means the uniqueness of the partition of the word $u$ with $|u| \geq L_{\omega}$ into blocks. 
An occurrence of a word $u \in \Sigma^{*}$ in the word $\omega$ is a pair $(u, m)$ such that $u=\omega_{m+1} \omega_{m+2} \ldots \omega_{m+n}$. It is easy to see that a word can have many different occurrences. Let $\omega=\varphi(\omega)$. An occurrence $(v, p)$ of a word $v$ of length $k$ is called the ancestor of an occurrence $(u, m)$ of the word $u$ if there exists an interpretation $\langle v, i, j\rangle$ of $u$ such that $m=p l+i$.

Remark 2 Let $\varphi$ be a marked morphism. Then by Remark 1 circularity of a word $\omega=\varphi(\omega)$ means that any of its subwords $u$ with $|u| \geq L_{\omega}$ admits a unique interpretation $\langle v, i, j\rangle$ on $\omega$. So if $u$ is a subword of $\omega$ with $|u| \geq L_{\omega}$, then interpretations of all occurrences of $u$ are the same and equal to $\langle v, i, j\rangle$.

Now we introduce the main object of this paper. Let $\mathcal{A}_{\mathbb{N}}$ be the set of all sequences of pairwise distinct reals defined on $\mathbb{N}=\{0,1,2, \ldots\}$. Define an equivalence relation $\sim$ on $\mathcal{A}_{\mathbb{N}}$ as follows: let $a, b$ be sequences from $\mathcal{A}_{\mathbb{N}}$, where $a=\left\{a_{s}\right\}_{s \in \mathbb{N}}$ and $b=\left\{b_{s}\right\}_{s \in \mathbb{N}}$; then $a \sim b$ if and only if for all $s, r \in \mathbb{N}$ the inequalities $a_{s}<a_{r}$ and $b_{s}<b_{r}$ hold or do not hold simultaneously. An equivalence class from $\mathcal{A}_{\mathbb{N}} / \sim$ is called an $(\mathbb{N})$-permutation, or a one-sided infinite permutation. If a permutation $\alpha$ is realized by a sequence of reals $a$, that is, if the sequence $a$ belongs to the class $\alpha$, we denote $\alpha=\bar{a}$. Similarly, we can consider a $\mathbb{Z}$-permutation, or an $S$-permutation, defined on an arbitrary subset $S$ of $\mathbb{Z}$. In particular, a $\{1, \ldots, n\}$-permutation always has a representative with all values in $\{1, \ldots, n\}$, i. e., can be identified with a usual permutation from $S_{n}$. In what follows the notation $x=x_{1} \ldots x_{n}$, where $x_{i} \in\{1, \ldots, n\}$, $x_{i} \neq x_{j}$ for $i \neq j$, means that the sequence $x_{1}, \ldots, x_{n}$ is a representative of the permutation $x$, that is, that $x=\overline{x_{1} \ldots x_{n}}$.

Let $\omega$ be an infinite nonperiodic word over the alphabet $\Sigma$. A word $\omega$ corresponds to the binary real number $R_{\omega}(i)=0, \omega_{i} \omega_{i+1} \ldots=\sum_{k \geq 0} \omega_{i+k} 2^{-(k+1)}$. We will say that the infinite permutation $\alpha_{\omega}$ is generated by $\omega$ if it is realized by the sequence of $\alpha_{i}=R_{\omega}(i)$.

Since $\omega$ is a non-periodic word, all $R_{\omega}(i)$ are distinct, and the definition above is correct.

We define a function $\gamma: \mathbb{R}^{2} \backslash\{(a, a) \mid a \in \mathbb{R}\} \rightarrow\{<,>\}$, which for two different real numbers reveals their relation: $\gamma(a, b)=<$ if and only if $a<b$. We say that a permutation $\pi=\pi_{1} \ldots \pi_{n}$ is a subpermutation or factor of length $n$ of an infinite permutation $\alpha_{\omega}$ if $\gamma\left(\pi_{s}, \pi_{t}\right)=\gamma\left(\alpha_{i+s}, \alpha_{i+t}\right)$ for $1 \leq s<t \leq n$ and for a fixed positive integer $i$. In this case we write that $\pi=\alpha_{i+1} \ldots \alpha_{i+n}$.

We define the set $\operatorname{Perm}(n)$ as the set of all subpermutations of $\alpha_{\omega}$ of length $n$ :

$$
\operatorname{Perm}(n)=\left\{\alpha_{i+1} \ldots \alpha_{i+n} \mid i \geq 0\right\}
$$

Now we define the permutation complexity of a word $\omega$ (or equivalently, the factor complexity of the induced permutation $\left.\alpha_{\omega}\right)$ as $\lambda(n)=|\operatorname{Perm}(n)|$. We say that an occurrence $(u, m)$ of a word $u$ of length $n$ generates a permutation $\pi=\pi(u, m)$ if $\alpha_{m+1} \ldots \alpha_{m+n}=\pi$. A subword $u$ of the word $\omega$ generates a permutation $\pi$ if there is an occurrence $(u, m)$ of this word which generates $\pi$. The number of permutations generated by $u$ is denoted by $f(u)$.

\section{General scheme}

The main idea of the proof is that we calculate $\sum_{|u|=n} f(u)$. We note that $\sum_{|u|=n} f(u)$ is the number of permutations, each of them generated by at least one occurrence of some subword of length $n$ of word $\omega$. It is clear that some permutations can be calculated several times. But in Makarov (2006) it was proved that two distinct subwords $u_{1}$ and $u_{2}$ of word $\omega$ can generate the same permutations if and only if $u_{1}=v 0$ and $u_{2}=v 1$ up to a relabeling of $u_{1}$ and $u_{2}$. Recall that a subword $v$ of the word $\omega$ is called special (in 
$\omega)$ if $v 0$ and $v 1$ are also subwords of $\omega$. The set of all the special words of length $n$ is denoted by $B(n)$. Thus permutation complexity of $\omega$ is

$$
\sum_{|u|=n} f(u)-\sum_{b \in B(n-1)} g(b)
$$

where $g(v)$ is the number of common permutations generated by some occurrences of words $v 0$ and $v 1$. After we prove that we can express $f(u)$ through $f\left(u^{\prime}\right)$, where $u^{\prime}$ is the ancestor of $u$. We also show that we can express $g(v)$ through $g\left(v^{\prime}\right)$, where $v^{\prime}$ is the ancestor of $v$ and $v$ is a special word. So we reduce the calculation of $\sum_{|u|=n} f(u)$ and $\sum_{b \in B(n-1)} g(b)$ to the calculation of $\sum_{|u|=k} f(u)$ and $\sum_{b \in B(k-1)} g(b)$, where $k$ is a sufficiently small number.

\section{Class $Q_{l}$}

We say that a $l$-uniform marked binary morphism $\varphi$ such that $\varphi(0)$ starts with 0 belongs to the class $Q_{l}$ if it satisfies the following properties (we assume that $l \geq 2$ ):

Properties.

1. If $\varphi(0)=0 u 0 x$ for some word $x$, then $0 u 1$ is not a subword of $\varphi(0)$ and $\varphi(1)$ and $0 u$ is not a suffix of $\varphi(0)$ and $\varphi(1)$.

2. If $\varphi(1)=1 u 1 x$ for some word $x$, then $1 u 0$ is not a subword of $\varphi(0)$ and $\varphi(1)$ and $1 u$ is not a suffix of $\varphi(0)$ and $\varphi(1)$.

In Frid (1998) the criterion of circularity of marked uniform morphisms was obtained. According to an easy corollary of that criterion Frid (2000), all nonperiodic fixed points of $l$-uniform binary morphisms with $\omega_{1}=0$ are circular, except for the case when $\varphi(1)=1^{l}$. So $\omega=\lim _{n \rightarrow \infty} \varphi^{n}(0)$ of any morphism $\varphi$ which belongs to the class $Q_{l}$ is circular because $\varphi(1) \neq 1^{l}$ (if $\varphi(1)=1^{l}$, then we obtain a contradiction with Property 2 in the definition of $Q_{l}$ ).

Everywhere below the word $\varphi(0)$ will be called the block of the first type, and $\varphi(1)$ is called the block of the second type.

Example. Each morphism $\varphi(0)=01^{2 n} 01^{n}, \varphi(1)=10^{2 n} 10^{n}$ for $n \geq 2$ belongs to $Q_{l}$, whereas the morphism $\varphi(0)=01011, \varphi(1)=10000$ does not belong to $Q_{l}$.

Consider a fixed point $\omega=\varphi(\omega)$ of a morphism $\varphi \in Q_{l}$. The partition of $\omega$ into blocks which are the images of its symbols is called correct.

Let $u$ and $v$ be two words such that $|u|=|v|$. We say that $u>v$ (or $v<u$ ) if $u=u_{1} 1 u_{2}$ and $v=u_{1} 0 v_{2}$ for some words $u_{1}, u_{2}$ and $v_{2}$.

Lemma 1 Let $\omega$ be a fixed point of the morphism $\varphi$, where $\varphi \in Q_{l}$. Then the following statements are true:

1. Let $\omega_{i}=\omega_{j}=0$ and $i \equiv 1(\bmod l), j \not \equiv 1(\bmod l)$. Then $R_{\omega}(i)>R_{\omega}(j)$.

2. Let $\omega_{i}=\omega_{j}=1$ and $i \equiv 1(\bmod l), j \not \equiv 1(\bmod l)$. Then $R_{\omega}(i)<R_{\omega}(j)$.

To prove Lemma1, we prove several auxiliary assertions. 
Proposition 1 Let $\varphi \in Q_{l}$. Then the following statements are true:

1. If $0 u$ is a prefix of $\varphi(0)$ and $0 v$ is a subword of $\varphi(0)$ or $\varphi(1)$ with $|u|=|v|$, then $v<u$ or $v=u$.

2. If $1 u$ is a prefix of $\varphi(1)$ and $1 v$ is a subword of $\varphi(0)$ or $\varphi(1)$ with $|u|=|v|$, then $v>u$ or $v=u$.

Proof: The two cases are symmetric, so we shall prove the first one. Let $v>u$. Hence $v=u_{1} 1 v_{2}$ and $u=u_{1} 0 u_{2}$ for some words $u_{1}, u_{2}$ and $v_{2}$. So $0 u_{1} 0$ is a prefix of $\varphi(0)$ and $0 u_{1} 1$ is a subword of $\varphi(0)$ or $\varphi(1)$. It contradicts with Property 1 of the definition of $Q_{l}$.

Proposition 2 Let $\omega$ be a some binary nonperiodic word. Let $R_{\omega}(i)=0, x u \ldots$ and $R_{\omega}(j)=0, x v \ldots$ for some finite words $x$, $u$ and $v$ such that $u>v$. Then $R_{\omega}(i)>R_{\omega}(j)$.

Proof: Since $u>v$, we have $u=u_{1} 1 u_{2}$ and $v=u_{1} 0 v_{2}$ for some words $u_{1}, u_{2}$ and $v_{2}$. So

$$
\gamma\left(R_{\omega}(i), R_{\omega}(j)\right)=\gamma\left(0, x u_{1} 1 \ldots ; 0, x u_{1} 0 \ldots\right)=>
$$

Proof of Lemma 1. The two cases are symmetric, so we shall prove the first one. Since $\omega_{i}=0$ and $i \equiv 1(\bmod l), \omega_{i}$ lies in block $\varphi(0)$ in the correct partition of $\omega$ into blocks. Let $\omega_{j}$ lie in block $\varphi(c)$ in the correct partition of $\omega$ into blocks for some $c \in\{0,1\}$. There exist words $u, u^{\prime}, x$ and $y$ such that $\varphi(0)=0 u y, \varphi(c)=x \omega_{j} u^{\prime}$ and $|u|=\left|u^{\prime}\right|$.

Proposition 1 implies that $u^{\prime}<u$ or $u^{\prime}=u$. Consider the case when $u^{\prime}<u$. Then we have

$$
\gamma\left(R_{\omega}(i), R_{\omega}(j)\right)=\gamma\left(0, \omega_{i} u \ldots ; 0, \omega_{j} u^{\prime} \ldots\right)=>
$$

due to Proposition 2 .

Consider the case when $u=u^{\prime}$. Then $\varphi(c)=x \omega_{j} u$. Let $\varphi(0)=0 u a v$ for some letter $a$ and word $v$. Since $0 u$ is a suffix of $\varphi(c)$, by Property 1 of the definition of $Q_{l}$ we have that $a=1$. So $\varphi(0)=0 u 1 v$. If $\omega_{j+|u|+1}=0$, then

$$
\gamma\left(R_{\omega}(i), R_{\omega}(j)\right)=\gamma\left(0, \omega_{i} u 1 \ldots ; 0, \omega_{j} u 0 \ldots\right)=>
$$

Consider the case when $\omega_{j+|u|+1}=1$. Since $\varphi(c)=x \omega_{j} u$, we have $j+|u|+1 \equiv 1(\bmod l)$ and $\omega_{j+|u|+1}$ lies in block $\varphi(1)$ in the correct partition $\omega$ into blocks. Let $\varphi(1)=1 v^{\prime} b s$, where $v^{\prime}$ and $s$ are some words and $b$ is a letter such that $|v|=\left|v^{\prime}\right|$. Hence $|s|=|u|$. By Proposition 1 we have $v^{\prime}<v$ or $v=v^{\prime}$. If $v^{\prime}<v$, then

$$
\gamma\left(R_{\omega}(i), R_{\omega}(j)\right)=\gamma\left(0, \omega_{i} u 1 v \ldots ; 0, \omega_{j} u 1 v^{\prime} \ldots\right)=>
$$

due to Proposition 2 So we can assume that $v=v^{\prime}$. Since $1 v$ is a suffix of $\varphi(0)$, by Property 2 of the definition of $Q_{l}$ we have that $b=0$. If $\omega_{i+l}=1$, then

$$
\gamma\left(R_{\omega}(i), R_{\omega}(j)\right)=\gamma\left(0, \omega_{i} u 1 v 1 \ldots ; 0, \omega_{j} u 1 v 0 \ldots\right)=>.
$$

So we can assume that $\omega_{i+l}=0$. Since $i \equiv 1(\bmod l)$, we have $i+l \equiv 1(\bmod l)$ and $\omega_{i+l}$ lies in block $\varphi(0)$ in the correct partition $\omega$ into blocks. By Proposition 1 we have $s<u$ or $s=u$. If $s<u$, then

$$
\gamma\left(R_{\omega}(i), R_{\omega}(j)\right)=\gamma\left(0, \omega_{i} u 1 v 0 u \ldots ; 0, \omega_{j} u 1 v 0 s \ldots\right)=>
$$


due to Proposition 2. So we can assume that $s=u$. If $\omega_{j+|u|+l+1}=0$, then

$$
\gamma\left(R_{\omega}(i), R_{\omega}(j)\right)=\gamma\left(0, \omega_{i} u 1 v 0 u 1 \ldots ; 0, \omega_{j} u 1 v 0 u 0 \ldots\right)=>.
$$

So we can assume that $\omega_{j+|u|+l+1}=1$.

Continuing the procedure, we obtain that either $R_{\omega}(i)>R_{\omega}(j)$, or $\omega=\omega_{1} \ldots \omega_{i-1} \varphi(0) \varphi(0) \ldots$ The second case is impossible because word $\omega$ is nonperiodic.

Lemma 2 Let $\omega$ be a fixed point of the morphism $\varphi$, where $\varphi \in Q_{l}$. Let $\omega_{i}=\omega_{j}$, where $i \equiv i^{\prime}(\bmod l)$, $j \equiv j^{\prime}(\bmod l)$ and $0 \leq i^{\prime}, j^{\prime} \leq l-1$. If $i^{\prime} \neq j^{\prime}$, or if $\omega_{i}$ and $\omega_{j}$ lie in blocks of different types in the correct partition of $\omega$ into blocks, then the relation $\gamma\left(R_{\omega}(i), R_{\omega}(j)\right)$ is uniquely defined by $i^{\prime}, j^{\prime}$ and the types of respective blocks.

Proof: Let $\omega_{i}$ and $\omega_{j}$ lie in blocks of different types in the correct partition of $\omega$ and $i^{\prime}=j^{\prime}$. Without loss of generality, we assume that $\omega_{i}$ lies in $\varphi(0)$ and $\omega_{j}$ lies in $\varphi(1)$. Then $\varphi(0)=x \omega_{i} y$ and $\varphi(1)=x^{\prime} \omega_{j} y^{\prime}$ where $|y|=\left|y^{\prime}\right|$. Since the last symbols of $y$ and $y^{\prime}$ are different, we have $y=z a v$ and $y^{\prime}=z b v^{\prime}$ where $a$ and $b$ are different symbols. Hence we obtain that

$$
\gamma\left(R_{\omega}(i), R_{\omega}(j)\right)=\gamma\left(0, \omega_{i} z a \ldots, 0, \omega_{j} z b \ldots\right)=\gamma(a, b) .
$$

In all other cases we have $i^{\prime} \neq j^{\prime}$. Without loss of generality, we assume that $i^{\prime}<j^{\prime}$. Let $T_{1}=x \omega_{i} y$ and $T_{2}=x^{\prime} \omega_{j} y^{\prime}$ be blocks which contain $\omega_{i}$ and $\omega_{j}$. Then there are two cases. In the first case we have $y=y^{\prime \prime} z$ where $\left|y^{\prime \prime}\right|=\left|y^{\prime}\right|$ and $y^{\prime \prime} \neq y^{\prime}$. Hence we obtain that $y^{\prime \prime}=g a g^{\prime \prime}, y^{\prime}=g b g^{\prime}$ where $g, g^{\prime}, g^{\prime \prime}$ are some words, $a$ and $b$ are different symbols. Hence we obtain that

$$
\gamma\left(R_{\omega}(i), R_{\omega}(j)\right)=\gamma\left(0, \omega_{i} g a \ldots, 0, \omega_{j} g b \ldots\right)=\gamma(a, b) .
$$

In the second case we have $y=y^{\prime} z$. Assume first that $z=0 z^{\prime}$ for some word $z^{\prime}$. If $\omega_{j+l-j^{\prime}+1}=0$, then Lemma 1 implies that $R_{\omega}\left(i+\left|y^{\prime}\right|+1\right)<R_{\omega}\left(j+l-j^{\prime}+1\right)$, because in this case $\omega_{j+l-j^{\prime}+1}=0$ and $j+l-j^{\prime}+1 \equiv 1(\bmod l)$. If $\omega_{j+l-j^{\prime}+1}=1$, then $R_{\omega}\left(i+\left|y^{\prime}\right|+1\right)<R_{\omega}\left(j+l-j^{\prime}+1\right)$. In addition, we have that

$$
\gamma\left(R_{\omega}(i), R_{\omega}(j)\right)=\gamma\left(0, \omega_{i} y^{\prime} 0 \ldots, 0, \omega_{j} y^{\prime} 0 \ldots\right)=\gamma\left(R_{\omega}\left(i+\left|y^{\prime}\right|+1\right), R_{\omega}\left(j+l-j^{\prime}+1\right)\right) .
$$

Thus, in this case the inequality $R_{\omega}(i)<R_{\omega}(j)$ always holds.

It remains to consider the case $z=1 z^{\prime}$. If $\omega_{j+l-j^{\prime}+1}=1$, then Lemma 1 implies that $R_{\omega}\left(i+\left|y^{\prime}\right|+1\right)>$ $R_{\omega}\left(j+l-j^{\prime}+1\right)$, because in this case $\omega_{j+l-j^{\prime}+1}=1$ and $j+l-j^{\prime}+1 \equiv 1(\bmod l)$. If $\omega_{j+l-j^{\prime}+1}=0$, then $R_{\omega}\left(i+\left|y^{\prime}\right|+1\right)>R_{\omega}\left(j+l-j^{\prime}+1\right)$. In addition, we have that

$$
\gamma\left(R_{\omega}(i), R_{\omega}(j)\right)=\gamma\left(0, \omega_{i} y^{\prime} 1 \ldots, 0, \omega_{j} y^{\prime} 1 \ldots\right)=\gamma\left(R_{\omega}\left(i+\left|y^{\prime}\right|+1\right), R_{\omega}\left(j+l-j^{\prime}+1\right)\right) .
$$

Thus, in this case the inequality $R_{\omega}(i)>R_{\omega}(j)$ always holds.

Lemma 3 Let $\left(u, m_{1}\right)$ and $\left(u, m_{2}\right)$ be two occurrences of a subword $u$ of length $n \geq L_{\omega},\left(u^{\prime}, m_{1}^{\prime}\right)$ and $\left(u^{\prime}, m_{2}^{\prime}\right)$ be their ancestors. Then for $1 \leq t<s \leq n$ either

$$
\gamma\left(R_{\omega}\left(m_{1}+t\right), R_{\omega}\left(m_{1}+s\right)\right)=\gamma\left(R_{\omega}\left(m_{2}+t\right), R_{\omega}\left(m_{2}+s\right)\right)
$$


or $m_{1}+t=\left(m_{1}^{\prime}+t^{\prime}-1\right) l+r, m_{1}+s=\left(m_{1}^{\prime}+s^{\prime}-1\right) l+r, m_{2}+t=\left(m_{2}^{\prime}+t^{\prime}-1\right) l+r$, $m_{2}+s=\left(m_{2}^{\prime}+s^{\prime}-1\right) l+r$ for some $1 \leq r \leq l$ and

$$
\omega_{m_{1}^{\prime}+t^{\prime}}=\omega_{m_{1}^{\prime}+s^{\prime}}=\omega_{m_{2}^{\prime}+t^{\prime}}=\omega_{m_{2}^{\prime}+s^{\prime}} .
$$

Proof: Let $1 \leq t<s \leq n$. Consider relations $\gamma\left(R_{\omega}\left(m_{1}+t\right), R_{\omega}\left(m_{1}+s\right)\right)$ and $\gamma\left(R_{\omega}\left(m_{2}+t\right), R_{\omega}\left(m_{2}+\right.\right.$ $s))$. If $\omega_{m_{1}+t} \neq \omega_{m_{1}+s}$ and $\omega_{m_{2}+t} \neq \omega_{m_{2}+s}$, then

$$
\gamma\left(R_{\omega}\left(m_{1}+t\right), R_{\omega}\left(m_{1}+s\right)\right)=\gamma\left(\omega_{m_{1}+t}, \omega_{m_{1}+s}\right)
$$

and

$$
\gamma\left(R_{\omega}\left(m_{2}+t\right), R_{\omega}\left(m_{2}+s\right)\right)=\gamma\left(\omega_{m_{2}+t}, \omega_{m_{2}+s}\right) .
$$

So

$$
\gamma\left(R_{\omega}\left(m_{1}+t\right), R_{\omega}\left(m_{1}+s\right)\right)=\gamma\left(R_{\omega}\left(m_{2}+t\right), R_{\omega}\left(m_{2}+s\right)\right) .
$$

Consider the case when $\omega_{m_{1}+t}=\omega_{m_{1}+s}$ and $\omega_{m_{2}+t}=\omega_{m_{2}+s}$. Let $u_{1}=\omega_{m_{1}+1} \ldots \omega_{m_{1}+n}$ and $u_{2}=$ $\omega_{m_{2}+1} \ldots \omega_{m_{2}+n}$. Since $\left|u_{1}\right|=\left|u_{2}\right| \geq L_{\omega}$, by Remark 1 words $u_{1}$ and $u_{2}$ have the same partitions in the correct partition of $\omega$. Hence $\omega_{m_{1}+t}, \omega_{m_{1}+s}, \omega_{m_{2}+t}$ and $\omega_{m_{2}+s}$ lie in blocks $\varphi\left(\omega_{m_{1}^{\prime}+t^{\prime}}\right), \varphi\left(\omega_{m_{1}^{\prime}+s^{\prime}}\right)$, $\varphi\left(\omega_{m_{2}^{\prime}+t^{\prime}}\right)$ and $\varphi\left(\omega_{m_{2}^{\prime}+s^{\prime}}\right)$ in the correct partition of $\omega$ for some $1 \leq t^{\prime}<s^{\prime} \leq\left|u^{\prime}\right|$. Moreover $\omega_{m_{1}+t}$ and $\omega_{m_{2}+t}$ occur in blocks $\varphi\left(\omega_{m_{1}^{\prime}+t^{\prime}}\right)$ and $\varphi\left(\omega_{m_{2}^{\prime}+t^{\prime}}\right)$ at the same positions. Analogously $\omega_{m_{1}+s}$ and $\omega_{m_{2}+s}$ occur in blocks $\varphi\left(\omega_{m_{1}^{\prime}+s^{\prime}}\right)$ and $\varphi\left(\omega_{m_{2}^{\prime}+s^{\prime}}\right)$ at the same positions. So $m_{1}+t \equiv m_{2}+t(\bmod l)$ and $m_{1}+s \equiv m_{2}+s(\bmod l)$. Moreover $\omega_{m_{1}^{\prime}+t^{\prime}}=\omega_{m_{2}^{\prime}+t^{\prime}}$ and $\omega_{m_{1}^{\prime}+s^{\prime}}=\omega_{m_{2}^{\prime}+s^{\prime}}$.

Let

$$
\omega_{m_{1}^{\prime}+t^{\prime}}=\omega_{m_{2}^{\prime}+t^{\prime}}=a
$$

and

$$
\omega_{m_{1}^{\prime}+s^{\prime}}=\omega_{m_{2}^{\prime}+s^{\prime}}=b .
$$

If $a \neq b$, then $\omega_{m_{1}+t}$ and $\omega_{m_{1}+s}$ (as $\omega_{m_{2}+t}$ and $\omega_{m_{2}+s}$ ) lie in blocks of different types in the correct partition of $\omega$ into blocks. Since $m_{1}+t \equiv m_{2}+t(\bmod l)$ and $m_{1}+s \equiv m_{2}+s(\bmod l)$, Lemma2 implies that

$$
\gamma\left(R_{\omega}\left(m_{1}+t\right), R_{\omega}\left(m_{1}+s\right)\right)=\gamma\left(R_{\omega}\left(m_{2}+t\right), R_{\omega}\left(m_{2}+s\right)\right) .
$$

If $a=b$ and $s \not \equiv t(\bmod l)$, then $m_{1}+t \not \equiv m_{1}+s(\bmod l), m_{2}+t \not \equiv m_{2}+s(\bmod l)$ and Lemma2 also implies that

$$
\gamma\left(R_{\omega}\left(m_{1}+t\right), R_{\omega}\left(m_{1}+s\right)\right)=\gamma\left(R_{\omega}\left(m_{2}+t\right), R_{\omega}\left(m_{2}+s\right)\right) .
$$

It remains to consider the case when $a=b$ and $s \equiv t(\bmod l)$. Then

$$
\omega_{m_{1}^{\prime}+t^{\prime}}=\omega_{m_{1}^{\prime}+s^{\prime}}=\omega_{m_{2}^{\prime}+t^{\prime}}=\omega_{m_{2}^{\prime}+s^{\prime}} .
$$

Moreover since $s \equiv t(\bmod l)$, we have $m_{1}+t=\left(m_{1}^{\prime}+t^{\prime}-1\right) l+r, m_{1}+s=\left(m_{1}^{\prime}+s^{\prime}-1\right) l+r$, $m_{2}+t=\left(m_{2}^{\prime}+t^{\prime}-1\right) l+r$ and $m_{2}+s=\left(m_{2}^{\prime}+s^{\prime}-1\right) l+r$ for some $1 \leq r \leq l$.

Lemma 4 Let $\omega_{i}=\omega_{j}$ and $R_{\omega}(i)<R_{\omega}(j)$. Then the inequalities $R_{\omega}((i-1) l+r)<R_{\omega}((j-1) l+r)$ hold for all $1 \leq r \leq l$. 
Proof: Since $\omega_{i}=\omega_{j}$, we obtain that $\varphi\left(\omega_{i}\right)=\varphi\left(\omega_{j}\right)$. Hence we have that the equality $\omega_{(i-1) l+r}=$ $\omega_{(j-1) l+r}$ holds for all $1 \leq r \leq l$. Since $R_{\omega}(i)<R_{\omega}(j)$ then there exists a finite binary word $x$ such that $R_{\omega}(i)=0, \omega_{i} x 0 \ldots$ and $R_{\omega}(j)=0, \omega_{j} x 1 \ldots$ Hence we have

$$
R_{\omega}((i-1) l+r)=0, \omega_{(i-1) l+r} \ldots \omega_{i l} \varphi(x) 0 \ldots
$$

and

$$
R_{\omega}((j-1) l+r)=0, \omega_{(j-1) l+r} \ldots \omega_{j l} \varphi(x) 1 \ldots
$$

Consequently we obtain that $R_{\omega}((i-1) l+r)<R_{\omega}((j-1) l+r)$. The lemma is proved.

Lemma 5 Let $\left(v 0, m_{1}\right)$ and $\left(v 1, m_{2}\right)$ be some occurrences of words $v 0$ and $v 1$ with $|v|=n \geq L_{\omega}$, and let $\left(v^{\prime} 0, m_{1}^{\prime}\right)$ and $\left(v^{\prime} 1, m_{2}^{\prime}\right)$ be their ancestors with $\left|v^{\prime}\right|=k$. Then the following statements are true:

1. If $m_{1}+t \not \equiv 1(\bmod l)$ and $m_{2}+t \not \equiv 1(\bmod l)$, then

$$
\gamma\left(R_{\omega}\left(m_{1}+t\right), R_{\omega}\left(m_{1}+n+1\right)\right)=\gamma\left(R_{\omega}\left(m_{2}+t\right), R_{\omega}\left(m_{2}+n+1\right)\right)
$$

for $1 \leq t<n+1$.

2. If $m_{1}+t=\left(m_{1}^{\prime}+t^{\prime}-1\right) l+1$ and $m_{2}+t=\left(m_{2}^{\prime}+t^{\prime}-1\right) l+1$ for $1 \leq t^{\prime}<k+1$ and $1 \leq t<n+1$, then

$$
\gamma\left(R_{\omega}\left(m_{1}+t\right), R_{\omega}\left(m_{1}+n+1\right)\right)=\gamma\left(R_{\omega}\left(m_{2}+t\right), R_{\omega}\left(m_{2}+n+1\right)\right)
$$

if and only if

$$
\gamma\left(R_{\omega}\left(m_{1}^{\prime}+t^{\prime}\right), R_{\omega}\left(m_{1}^{\prime}+k+1\right)\right)=\gamma\left(R_{\omega}\left(m_{2}^{\prime}+t^{\prime}\right), R_{\omega}\left(m_{2}^{\prime}+k+1\right)\right) .
$$

Proof: Let $1 \leq t<n+1$. Let $v_{1}=\omega_{m_{1}+1} \ldots \omega_{m_{1}+n}$ and $v_{2}=\omega_{m_{2}+1} \ldots \omega_{m_{2}+n}$. Since $\left|v_{1}\right|=\left|v_{2}\right| \geq$ $L_{\omega}$, by Remark 1 words $v_{1}$ and $v_{2}$ have the same partitions in the correct partition of $\omega$. Hence $\omega_{m_{1}+t}$ and $\omega_{m_{2}+t}$ lie in blocks $\varphi\left(\omega_{m_{1}^{\prime}+t^{\prime}}\right)$ and $\varphi\left(\omega_{m_{2}^{\prime}+t^{\prime}}\right)$ in the correct partition of $\omega$ for some $1 \leq t^{\prime} \leq k$. Moreover $\omega_{m_{1}+t}$ and $\omega_{m_{2}+t}$ occur in blocks $\varphi\left(\omega_{m_{1}^{\prime}+t^{\prime}}\right)$ and $\varphi\left(\omega_{m_{2}^{\prime}+t^{\prime}}\right)$ at the same positions. So $m_{1}+t \equiv m_{2}+t$ $(\bmod l)$. Since $v$ is a special word, $v$ has the interpretation $\left\langle v^{\prime}, i, 0\right\rangle$, where $0 \leq i<l$. Hence the last blocks of partitions of $v_{1}$ and $v_{2}$ in the correct partition of $\omega$ are complete. So $m_{1}+n+1 \equiv 1(\bmod l)$ and $m_{2}+n+1 \equiv 1(\bmod l)$. Since $\omega_{m_{1}+n+1}=0$ and $\omega_{m_{2}+n+1}=1$, we have that $\omega_{m_{1}+n+1}$ and $\omega_{m_{2}+n+1}$ are the first symbols of blocks $\varphi\left(\omega_{m_{1}^{\prime}+k+1}\right)=\varphi(0)$ and $\varphi\left(\omega_{m_{2}^{\prime}+k+1}\right)=\varphi(1)$ in the correct partition of $\omega$. Hence $m_{1}+n+1=\left(m_{1}^{\prime}+k\right) l+1, m_{2}+n+1=\left(m_{2}^{\prime}+k\right) l+1$ and $\omega_{m_{1}^{\prime}+k+1}=0$, $\omega_{m_{2}^{\prime}+k+1}=1$.

Consider the case when $m_{1}+t \not \equiv 1(\bmod l)$ and $m_{2}+t \not \equiv 1(\bmod l)$. Assume that

$$
\omega_{m_{1}+t}=\omega_{m_{2}+t}=0
$$

(the other case is similar). Recall that $\omega_{m_{1}+n+1}=0$. Since $m_{1}+t \not \equiv 1(\bmod l)$ and $m_{1}+n+1 \equiv 1$ $(\bmod l)$, we have that $R_{\omega}\left(m_{1}+t\right)<R_{\omega}\left(m_{1}+n+1\right)$ due to Lemma 1 Since $\omega_{m_{2}+t}=0$ and $\omega_{m_{2}+n+1}=1$, we have that

$$
\gamma\left(R_{\omega}\left(m_{2}+t\right), R_{\omega}\left(m_{2}+n+1\right)\right)=\gamma(0,0 \ldots ; 0,1 \ldots)=<.
$$


So

$$
\gamma\left(R_{\omega}\left(m_{1}+t\right), R_{\omega}\left(m_{1}+n+1\right)\right)=\gamma\left(R_{\omega}\left(m_{2}+t\right), R_{\omega}\left(m_{2}+n+1\right)\right) .
$$

Consider the case when $m_{1}+t \equiv 1(\bmod l)$ and $m_{2}+t \equiv 1(\bmod l)$. As we note above $\omega_{m_{1}+t}$ and $\omega_{m_{2}+t}$ lie in blocks $\varphi\left(\omega_{m_{1}^{\prime}+t^{\prime}}\right)$ and $\varphi\left(\omega_{m_{2}^{\prime}+t^{\prime}}\right)$ in the correct partition of $\omega$ for some $1 \leq t^{\prime}<k+1$. Hence $m_{1}+t=\left(m_{1}^{\prime}+t^{\prime}-1\right) l+1$ and $m_{2}+t=\left(m_{2}^{\prime}+t^{\prime}-1\right) l+1$. Assume that

$$
\omega_{m_{1}+t}=\omega_{m_{2}+t}=0
$$

(the case when $\omega_{m_{1}+t}=\omega_{m_{2}+t}=1$ is similar). Since $\varphi(0)$ starts with 0 and $\varphi(1)$ starts with 1 , we have that $\omega_{m_{1}^{\prime}+t^{\prime}}=\omega_{m_{2}^{\prime}+t^{\prime}}=0$. Recall that $\omega_{m_{2}+n+1}=\omega_{m_{2}^{\prime}+k+1}=1$. Then

$$
\gamma\left(R_{\omega}\left(m_{2}+t\right), R_{\omega}\left(m_{2}+n+1\right)\right)=\gamma\left(R_{\omega}\left(m_{2}^{\prime}+t^{\prime}\right), R_{\omega}\left(m_{2}^{\prime}+k+1\right)\right) .
$$

Lemma 4 implies that

$$
\gamma\left(R_{\omega}\left(m_{1}+t\right), R_{\omega}\left(m_{1}+n+1\right)\right)=\gamma\left(R_{\omega}\left(m_{1}^{\prime}+t^{\prime}\right), R_{\omega}\left(m_{1}^{\prime}+k+1\right)\right) .
$$

So

$$
\gamma\left(R_{\omega}\left(m_{1}+t\right), R_{\omega}\left(m_{1}+n+1\right)\right)=\gamma\left(R_{\omega}\left(m_{2}+t\right), R_{\omega}\left(m_{2}+n+1\right)\right)
$$

if and only if

$$
\gamma\left(R_{\omega}\left(m_{1}^{\prime}+t^{\prime}\right), R_{\omega}\left(m_{1}^{\prime}+k+1\right)\right)=\gamma\left(R_{\omega}\left(m_{2}^{\prime}+t^{\prime}\right), R_{\omega}\left(m_{2}^{\prime}+k+1\right)\right) .
$$

\section{Conjugacy of permutations}

In this section we introduce the concept of conjugate permutations. Let $z=z_{1} z_{2} \ldots z_{k}$ be a permutation of length $k$, where $z_{i} \in\{1,2, \ldots, k\}$.

An element of the permutation $z$ is the number $z_{i}$, where $1 \leq i \leq k$.

We will say that two permutations $x=x_{1} x_{2} \ldots x_{k}$ and $y=y_{1} y_{2} \ldots y_{k}$ are conjugate if they differ only in relations of extreme elements, i.e $\gamma\left(x_{1}, x_{k}\right) \neq \gamma\left(y_{1}, y_{k}\right)$, but $\gamma\left(x_{i}, x_{j}\right)=\gamma\left(y_{i}, y_{j}\right)$ for all other $i, j$. We will denote this conjugacy by $x \sim y$.

Example. There are exactly two pairs of conjugate permutations among the permutations of length 3 : $132 \sim 231$ and $213 \sim 312$.

Lemma 6 Let $x$ be a finite permutation and $x=x_{1} \ldots x_{k}$. Then a permutation $y$ such that $x \sim y$ exists if and only if $\left|x_{1}-x_{k}\right|=1$.

Proof: Let us prove the necessity. Let $y$ be a permutation such that $x \sim y$. Let us prove that $\left|x_{1}-x_{k}\right|=1$. If it is not true, then there exists $1<i<k$ such that $\min \left(x_{1}, x_{k}\right)<x_{i}<\max \left(x_{1}, x_{k}\right)$. Since $x \sim y$, we obtain that $\gamma\left(x_{1}, x_{i}\right)=\gamma\left(y_{1}, y_{i}\right)$ and $\gamma\left(x_{i}, x_{k}\right)=\gamma\left(y_{i}, y_{k}\right)$. Hence $\gamma\left(x_{1}, x_{k}\right)=\gamma\left(y_{1}, y_{k}\right)$. It contradicts with $x \sim y$.

Let us prove the sufficiency. Let $\left|x_{1}-x_{k}\right|=1$. We construct a permutation $y$ as follows: let $y_{1}=x_{k}$, $y_{k}=x_{1}, y_{2}=x_{2}, y_{3}=x_{3} \ldots, y_{k-1}=x_{k-1}$. Since $\left|x_{1}-x_{k}\right|=1$, we obtain that $\gamma\left(x_{i}, x_{j}\right)=\gamma\left(y_{i}, y_{j}\right)$ for all $i, j$ except $i=1, j=k$. On the other hand, we have by construction that $\gamma\left(x_{1}, x_{k}\right) \neq \gamma\left(y_{1}, y_{k}\right)$, that is $x \sim y$. 
Lemma 7 Let $\left(u, m_{1}\right)$ and $\left(u, m_{2}\right)$ be two occurrences of a subword $u$ of length $n \geq L_{\omega}$, and $\left(u^{\prime}, m_{1}^{\prime}\right)$ and $\left(u^{\prime}, m_{2}^{\prime}\right)$ be their ancestors with $\left|u^{\prime}\right|=k$. Then the following statements are true:

1. If $\pi\left(u, m_{1}\right) \neq \pi\left(u, m_{2}\right)$, then $\pi\left(u^{\prime}, m_{1}^{\prime}\right) \neq \pi\left(u^{\prime}, m_{2}^{\prime}\right)$.

2. If $\pi\left(u, m_{1}\right) \sim \pi\left(u, m_{2}\right)$, then $\pi\left(u^{\prime}, m_{1}^{\prime}\right) \sim \pi\left(u^{\prime}, m_{2}^{\prime}\right)$ and $n=(k-1) l+1$.

3. If $\pi\left(u^{\prime}, m_{1}^{\prime}\right) \neq \pi\left(u^{\prime}, m_{2}^{\prime}\right)$ and $\pi\left(u^{\prime}, m_{1}^{\prime}\right) \nsim \pi\left(u^{\prime}, m_{2}^{\prime}\right)$, then $\pi\left(u, m_{1}\right) \neq \pi\left(u, m_{2}\right)$ and $\pi\left(u, m_{1}\right) \nsim$ $\pi\left(u, m_{2}\right)$.

4. If $n=(k-1) l+1$ and $\pi\left(u^{\prime}, m_{1}^{\prime}\right) \sim \pi\left(u^{\prime}, m_{2}^{\prime}\right)$, then $\pi\left(u, m_{1}\right) \sim \pi\left(u, m_{2}\right)$.

5. If $n<(k-1) l+1$ and $\pi\left(u^{\prime}, m_{1}^{\prime}\right) \sim \pi\left(u^{\prime}, m_{2}^{\prime}\right)$, then $\pi\left(u, m_{1}\right)=\pi\left(u, m_{2}\right)$.

6. If $n>(k-1) l+1$ and $\pi\left(u^{\prime}, m_{1}^{\prime}\right) \sim \pi\left(u^{\prime}, m_{2}^{\prime}\right)$, then $\pi\left(u, m_{1}\right) \neq \pi\left(u, m_{2}\right)$.

Proof: Let $u$ have the interpretation $\left\langle u^{\prime}, i, j\right\rangle$, where $0 \leq i<l, 0 \leq j<l$. It is unique since $|u| \geq L_{\omega}$.

1. Consider $1 \leq t<s \leq n$. Lemma3 3 implies that either

$$
\gamma\left(R_{\omega}\left(m_{1}+t\right), R_{\omega}\left(m_{1}+s\right)\right)=\gamma\left(R_{\omega}\left(m_{2}+t\right), R_{\omega}\left(m_{2}+s\right)\right)
$$

or $m_{1}+t=\left(m_{1}^{\prime}+t^{\prime}-1\right) l+r, m_{1}+s=\left(m_{1}^{\prime}+s^{\prime}-1\right) l+r, m_{2}+t=\left(m_{2}^{\prime}+t^{\prime}-1\right) l+r$, $m_{2}+s=\left(m_{2}^{\prime}+s^{\prime}-1\right) l+r$ for some $1 \leq r \leq l$ and

$$
\omega_{m_{1}^{\prime}+t^{\prime}}=\omega_{m_{1}^{\prime}+s^{\prime}}=\omega_{m_{2}^{\prime}+t^{\prime}}=\omega_{m_{2}^{\prime}+s^{\prime}} .
$$

Consider the second case. Then Lemma 4 implies that

$$
\gamma\left(R_{\omega}\left(m_{1}+t\right), R_{\omega}\left(m_{1}+s\right)\right)=\gamma\left(R_{\omega}\left(m_{1}^{\prime}+t^{\prime}\right), R_{\omega}\left(m_{1}^{\prime}+s^{\prime}\right)\right)
$$

and

$$
\gamma\left(R_{\omega}\left(m_{2}+t\right), R_{\omega}\left(m_{2}+s\right)\right)=\gamma\left(R_{\omega}\left(m_{2}^{\prime}+t^{\prime}\right), R_{\omega}\left(m_{2}^{\prime}+s^{\prime}\right)\right) .
$$

Since $\pi\left(u, m_{1}\right) \neq \pi\left(u, m_{2}\right)$, we obtain that

$$
\gamma\left(R_{\omega}\left(m_{1}+t\right), R_{\omega}\left(m_{1}+s\right)\right) \neq \gamma\left(R_{\omega}\left(m_{2}+t\right), R_{\omega}\left(m_{2}+s\right)\right)
$$

for some $1 \leq t<s \leq n$. Then we have

$$
\gamma\left(R_{\omega}\left(m_{1}^{\prime}+t^{\prime}\right), R_{\omega}\left(m_{1}^{\prime}+s^{\prime}\right)\right) \neq \gamma\left(R_{\omega}\left(m_{2}^{\prime}+t^{\prime}\right), R_{\omega}\left(m_{2}^{\prime}+s^{\prime}\right)\right)
$$

for the corresponding $t^{\prime}$ and $s^{\prime}$. Hence we prove that $\pi\left(u^{\prime}, m_{1}^{\prime}\right) \neq \pi\left(u^{\prime}, m_{2}^{\prime}\right)$.

2. Since $\left(u^{\prime}, m_{1}^{\prime}\right)$ is the ancestor of $\left(u, m_{1}\right)$, we obtain that $m_{1}+1=m_{1}^{\prime} l+i+1$ and $m_{1}+n=$ $\left(m_{1}^{\prime}+k\right) l-j$. So $n=k l-i-j$. Since $\pi\left(u, m_{1}\right) \sim \pi\left(u, m_{2}\right)$, we have

$$
\gamma\left(R_{\omega}\left(m_{1}+1\right), R_{\omega}\left(m_{1}+n\right)\right) \neq \gamma\left(R_{\omega}\left(m_{2}+1\right), R_{\omega}\left(m_{2}+n\right)\right) .
$$

Hence Lemma 3 implies that $m_{1}+1=m_{1}^{\prime} l+r, m_{1}+n=\left(m_{1}^{\prime}+k-1\right) l+r, m_{2}+1=m_{2}^{\prime} l+r$, $m_{2}+n=\left(m_{2}^{\prime}+k-1\right) l+r$ for some $1 \leq r \leq l$ and

$$
\omega_{m_{1}^{\prime}+1}=\omega_{m_{1}^{\prime}+k}=\omega_{m_{2}^{\prime}+1}=\omega_{m_{2}^{\prime}+k} .
$$


Hence $r=i+1=l-j$ and $n=k l-j-i=(k-1) l+1$. Lemma4 implies that

$$
\gamma\left(R_{\omega}\left(m_{1}+1\right), R_{\omega}\left(m_{1}+n\right)\right)=\gamma\left(R_{\omega}\left(m_{1}^{\prime}+1\right), R_{\omega}\left(m_{1}^{\prime}+k\right)\right)
$$

and

$$
\gamma\left(R_{\omega}\left(m_{2}+1\right), R_{\omega}\left(m_{2}+n\right)\right)=\gamma\left(R_{\omega}\left(m_{2}^{\prime}+1\right), R_{\omega}\left(m_{2}^{\prime}+k\right)\right)
$$

So

$$
\gamma\left(R_{\omega}\left(m_{1}^{\prime}+1\right), R_{\omega}\left(m_{1}^{\prime}+k\right)\right) \neq \gamma\left(R_{\omega}\left(m_{2}^{\prime}+1\right), R_{\omega}\left(m_{2}^{\prime}+k\right)\right) .
$$

Since $\pi\left(u, m_{1}\right) \sim \pi\left(u, m_{2}\right)$, all other relations between elements of $\pi\left(u, m_{1}\right)$ are equal to those of $\pi\left(u, m_{2}\right)$. If $\omega_{m_{1}^{\prime}+t^{\prime}} \neq \omega_{m_{1}^{\prime}+s^{\prime}}$ for some $1 \leq t^{\prime}<s^{\prime} \leq k$, then it is clear that

$$
\gamma\left(R_{\omega}\left(m_{1}^{\prime}+t^{\prime}\right), R_{\omega}\left(m_{1}^{\prime}+s^{\prime}\right)\right)=\gamma\left(R_{\omega}\left(m_{2}^{\prime}+t^{\prime}\right), R_{\omega}\left(m_{2}^{\prime}+s^{\prime}\right)\right) .
$$

By Lemma 4 the same equality holds when $\omega_{m_{1}^{\prime}+t^{\prime}}=\omega_{m_{1}^{\prime}+s^{\prime}}$ for some $1 \leq t^{\prime}<s^{\prime} \leq k$ with $t^{\prime} \neq 1$ or $s^{\prime} \neq k$. Since

$$
\gamma\left(R_{\omega}\left(m_{1}^{\prime}+1\right), R_{\omega}\left(m_{1}^{\prime}+k\right)\right) \neq \gamma\left(R_{\omega}\left(m_{2}^{\prime}+1\right), R_{\omega}\left(m_{2}^{\prime}+k\right)\right),
$$

we have proved that $\pi\left(u^{\prime}, m_{1}^{\prime}\right) \sim \pi\left(u^{\prime}, m_{2}^{\prime}\right)$.

3. Since $\pi\left(u^{\prime}, m_{1}^{\prime}\right) \neq \pi\left(u^{\prime}, m_{2}^{\prime}\right)$ and $\pi\left(u^{\prime}, m_{1}^{\prime}\right) \nsim \pi\left(u^{\prime}, m_{2}^{\prime}\right)$, we obtain that

$$
\gamma\left(R_{\omega}\left(m_{1}^{\prime}+t^{\prime}\right), R_{\omega}\left(m_{1}^{\prime}+s^{\prime}\right)\right) \neq \gamma\left(R_{\omega}\left(m_{2}^{\prime}+t^{\prime}\right), R_{\omega}\left(m_{2}^{\prime}+s^{\prime}\right)\right)
$$

for some $1 \leq t^{\prime}<s^{\prime} \leq k$ with $t^{\prime} \neq 1$ or $s^{\prime} \neq k$. Without loss of generality suppose that $s^{\prime}<k$ : say $R_{\omega}\left(m_{1}^{\prime}+t^{\prime}\right)<R_{\omega}\left(m_{1}^{\prime}+s^{\prime}\right)$ and $R_{\omega}\left(m_{2}^{\prime}+t^{\prime}\right)>R_{\omega}\left(m_{2}^{\prime}+s^{\prime}\right)$. Since

$$
\omega_{m_{1}^{\prime}+t^{\prime}}=u_{t^{\prime}}^{\prime}=\omega_{m_{2}^{\prime}+t^{\prime}}
$$

and

$$
\omega_{m_{1}^{\prime}+s^{\prime}}=u_{s^{\prime}}^{\prime}=\omega_{m_{2}^{\prime}+s^{\prime}},
$$

we obtain $\omega_{m_{1}^{\prime}+t^{\prime}}=\omega_{m_{1}^{\prime}+s^{\prime}}$ and $\omega_{m_{2}^{\prime}+t^{\prime}}=\omega_{m_{2}^{\prime}+s^{\prime}}$.

Then Lemma 4 implies that $R_{\omega}\left(\left(m_{1}^{\prime}+t^{\prime}\right) l\right)<R_{\omega}\left(\left(m_{1}^{\prime}+s^{\prime}\right) l\right)$ and $R_{\omega}\left(\left(m_{2}^{\prime}+t^{\prime}\right) l\right)>R_{\omega}\left(\left(m_{2}^{\prime}+\right.\right.$ $\left.\left.s^{\prime}\right) l\right)$. Since $s^{\prime} l<n$, we obtain that $\omega_{\left(m_{1}^{\prime}+s^{\prime}\right) l}$ and $\omega_{\left(m_{2}^{\prime}+s^{\prime}\right) l}$ are not the last symbols of the words $\omega_{m_{1}+1} \ldots \omega_{m_{1}+n}$ and $\omega_{m_{2}+1} \ldots \omega_{m_{2}+n}$, so $\pi\left(u, m_{1}\right) \neq \pi\left(u, m_{2}\right)$ and $\pi\left(u, m_{1}\right) \nsim \pi\left(u, m_{2}\right)$.

4. Assume that

$$
\gamma\left(R_{\omega}\left(m_{1}+t\right), R_{\omega}\left(m_{1}+s\right)\right) \neq \gamma\left(R_{\omega}\left(m_{2}+t\right), R_{\omega}\left(m_{2}+s\right)\right)
$$

for some $1 \leq t<s \leq n$. Let us prove that $t=1$ and $s=n$. Lemma 3 implies that $m_{1}+t=$ $\left(m_{1}^{\prime}+t^{\prime}-1\right) l+r, m_{1}+s=\left(m_{1}^{\prime}+s^{\prime}-1\right) l+r, m_{2}+t=\left(m_{2}^{\prime}+t^{\prime}-1\right) l+r, m_{2}+s=\left(m_{2}^{\prime}+s^{\prime}-1\right) l+r$ for some $1 \leq r \leq l$ and

$$
\omega_{m_{1}^{\prime}+t^{\prime}}=\omega_{m_{1}^{\prime}+s^{\prime}}=\omega_{m_{2}^{\prime}+t^{\prime}}=\omega_{m_{2}^{\prime}+s^{\prime}}
$$

Hence

$$
\gamma\left(R_{\omega}\left(m_{1}+t\right), R_{\omega}\left(m_{1}+s\right)\right)=\gamma\left(R_{\omega}\left(m_{1}^{\prime}+t^{\prime}\right), R_{\omega}\left(m_{1}^{\prime}+s^{\prime}\right)\right)
$$

and

$$
\gamma\left(R_{\omega}\left(m_{2}+t\right), R_{\omega}\left(m_{2}+s\right)\right)=\gamma\left(R_{\omega}\left(m_{2}^{\prime}+t^{\prime}\right), R_{\omega}\left(m_{2}^{\prime}+s^{\prime}\right)\right)
$$


due to Lemma 4 So

$$
\gamma\left(R_{\omega}\left(m_{1}^{\prime}+t^{\prime}\right), R_{\omega}\left(m_{1}^{\prime}+s^{\prime}\right)\right) \neq \gamma\left(R_{\omega}\left(m_{2}^{\prime}+t^{\prime}\right), R_{\omega}\left(m_{2}^{\prime}+s^{\prime}\right)\right) .
$$

Since $\pi\left(u^{\prime}, m_{1}^{\prime}\right) \sim \pi\left(u^{\prime}, m_{2}^{\prime}\right)$, we see that $t^{\prime}=1$ and $s^{\prime}=k$. Hence $s-t=\left(s^{\prime}-t^{\prime}\right) l=(k-1) l=n-1$. So $t=1$ and $s=n$.

Let us prove

$$
\gamma\left(R_{\omega}\left(m_{1}+1\right), R_{\omega}\left(m_{1}+n\right)\right) \neq \gamma\left(R_{\omega}\left(m_{2}+1\right), R_{\omega}\left(m_{2}+n\right)\right)
$$

Since $\left(u^{\prime}, m_{1}^{\prime}\right)$ is the ancestor of $\left(u, m_{1}\right)$, we obtain that $m_{1}+1=m_{1}^{\prime} l+i+1$ for some integer $i$. Analogously we obtain $m_{2}+1=m_{2}^{\prime} l+i+1$. Since $n=(k-1) l+1$, we have $m_{1}+n=\left(m_{1}^{\prime}+k-1\right) l+i+1$ and $m_{2}+n=\left(m_{2}^{\prime}+k-1\right) l+i+1$ for the same $i$ by Remark 2 as $n \geq L_{\omega}$. Then Lemma 4 implies that

$$
\gamma\left(R_{\omega}\left(m_{1}+1\right), R_{\omega}\left(m_{1}+n\right)\right)=\gamma\left(R_{\omega}\left(m_{1}^{\prime}+1\right), R_{\omega}\left(m_{1}^{\prime}+k\right)\right)
$$

and

$$
\gamma\left(R_{\omega}\left(m_{2}+t\right), R_{\omega}\left(m_{2}+s\right)\right)=\gamma\left(R_{\omega}\left(m_{2}^{\prime}+1\right), R_{\omega}\left(m_{2}^{\prime}+k\right)\right)
$$

So

$$
\gamma\left(R_{\omega}\left(m_{1}+1\right), R_{\omega}\left(m_{1}+n\right)\right) \neq \gamma\left(R_{\omega}\left(m_{2}+1\right), R_{\omega}\left(m_{2}+n\right)\right) .
$$

Thus $\pi\left(u, m_{1}\right) \sim \pi\left(u, m_{2}\right)$.

5. Since $u$ has the interpretation $\left\langle u^{\prime}, i, j\right\rangle, u$ is obtained from $\varphi\left(u^{\prime}\right)$ by erasing $i$ symbols to the left and $j$ symbols to the right. So $|u|=\left|u^{\prime}\right| l-i-j=k l-i-j$. Hence $0 \leq n+j-(k-1) l-1<l$. Let

$$
u_{1}=\omega_{m_{1}+n+1} \ldots \omega_{m_{1}+(k-1) l+1}
$$

and

$$
u_{2}=\omega_{m_{2}+n+1} \ldots \omega_{m_{2}+(k-1) l+1}
$$

(since $n<(k-1) l+1$, the definition of words $u_{1}$ and $u_{2}$ is correct). Then words $u u_{1}$ and $u u_{2}$ are obtained from $\varphi\left(u^{\prime}\right)$ by erasing $i$ symbols to the left and $n+j-(k-1) l-1$ symbols to the right. So $u u_{1}$ and $u u_{2}$ have the interpretation $\left\langle u^{\prime}, i, n+j-(k-1) l-1\right\rangle$ and $u_{1}=u_{2}$. Then $\left(u^{\prime}, m_{1}^{\prime}\right)$ and $\left(u^{\prime}, m_{2}^{\prime}\right)$ are the ancestors of occurrences $\left(u u_{1}, m_{1}\right)$ and $\left(u u_{2}, m_{2}\right)$. Since $\left|u u_{1}\right|=\left|u u_{2}\right|=\left(\left|u^{\prime}\right|-1\right) l+1$, Case 4 of this Lemma implies that $\pi\left(u u_{1}, m_{1}\right) \sim \pi\left(u u_{2}, m_{2}\right)$. Hence $\pi\left(u u_{1}, m_{1}\right)$ and $\pi\left(u u_{2}, m_{2}\right)$ differ only in relations of extreme elements and $\pi\left(u, m_{1}\right)=\pi\left(u, m_{2}\right)$.

6. Consider prefixes $u_{1}=\omega_{m_{1}+1} \ldots \omega_{m_{1}+(k-1) l+1}$ and $u_{2}=\omega_{m_{2}+1} \ldots \omega_{m_{2}+(k-1) l+1}$ of $u$. Since $u$ has the interpretation $\left\langle u^{\prime}, i, j\right\rangle, u$ is obtained from $\varphi\left(u^{\prime}\right)$ by erasing $i$ symbols to the left and $j$ symbols to the right. So $|u|=\left|u^{\prime}\right| l-i-j=k l-i-j$. Hence $0 \leq n+j-(k-1) l-1<l$. Then words $u_{1}$ and $u_{2}$ are obtained from $\varphi\left(u^{\prime}\right)$ by erasing $i$ symbols to the left and $n+j-(k-1) l-1$ symbols to the right. So $u_{1}$ and $u_{2}$ have the interpretation $\left\langle u^{\prime}, i, n+j-(k-1) l-1\right\rangle$. So $\left(u^{\prime}, m_{1}^{\prime}\right)$ and $\left(u^{\prime}, m_{2}^{\prime}\right)$ are the ancestors of occurrences $\left(u_{1}, m_{1}\right)$ and $\left(u_{2}, m_{2}\right)$, and $\left|u_{1}\right|=\left|u_{2}\right|=\left(\left|u^{\prime}\right|-1\right) l+1$. Then Case 4 of this Lemma implies that $\pi\left(u_{1}, m_{1}\right) \sim \pi\left(u_{2}, m_{2}\right)$. Hence we have

$$
\gamma\left(R_{\omega}\left(m_{1}+1\right), R_{\omega}\left(m_{1}+(k-1) l+1\right)\right) \neq \gamma\left(R_{\omega}\left(m_{2}+1\right), R_{\omega}\left(m_{2}+(k-1) l+1\right)\right) .
$$

Thus we prove that $\pi\left(u, m_{1}\right) \neq \pi\left(u, m_{2}\right)$. 


\section{Algorithm for finding $f(u)$}

Let $u$ be a subword of of length $n$ of $\omega$ and $n \geq L_{\omega}$. The number of permutations generated by $u$ is denoted by $f(u)$. In this section, we calculate $\sum_{|u|=n} f(u)$.

Proposition 3 Let $\varphi \in Q_{l}$ and $\omega=\varphi(\omega)$. Then $L_{\omega} \leq l^{2}+1$.

Proof: Since $\varphi$ is a marked uniform morphism, it is sufficient to prove the uniqueness of the partition of the word $u$ with $|u| \geq l^{2}+1$ into blocks. Let word $u$ have two distinct partitions into blocks, that is $u=s_{1} \varphi(x) p_{1}=s_{2} \varphi(y) p_{2}$, where $x$ and $y$ are some words, $s_{1}$ and $s_{2}$ are suffixes of $\varphi(a)$ and $\varphi(c)$ for $a, c \in\{0,1\}, p_{1}$ and $p_{2}$ are prefixes of $\varphi(b)$ and $\varphi(d)$ for $b, d \in\{0,1\}$. Let us prove that $|u| \leq l^{2}$. Let $x=x_{1} \ldots x_{t}$ and $y=y_{1} \ldots y_{h}$. Without loss of generality, we assume that $\left|s_{2}\right|<\left|s_{1}\right|$. Then $s_{1}=s_{2} e u$ for some word $u$ and letter $e$. Let $e=0$ (the case when $e=1$ is similar). Then $0 u$ is a suffix of $\varphi(a)$ and $0 u$ is a prefix of $\varphi\left(y_{1}\right)$. So $y_{1}=0$. Hence by Property 1 in the definition of $Q_{l}$ we have that $\varphi(0)=0 u 1 v$ for some word $v$. Then $\varphi\left(x_{1}\right)=1 v \ldots$ So $x_{1}=1$. Hence by the Property 1 in the definition of $Q_{l}$ we have that $\varphi(1)=1 v 0 u^{\prime}$. Then $\varphi\left(y_{2}\right)=0 \ldots$ and $y_{2}=0$. So $\varphi\left(y_{2}\right)=0 u 1 v$. Hence $u^{\prime}=u$ and $\varphi(1)=1 v 0 u$. Continuing the procedure, we obtain that $a=x_{1}=x_{2}=\ldots=x_{t}=b=1$. Then word $a x b=0^{t+2}$ is a subword of $\omega$. By the definition of $Q_{l}$ we have that $0^{l+1}$ and $1^{l+1}$ are not subwords of $\omega$. So $t \leq l-2$. Then $|u|=\left|s_{1}\right|+\left|p_{1}\right|+t l \leq l^{2}\left(\left|s_{1}\right| \leq l\right.$ and $\left|p_{1}\right| \leq l$ since $s_{1}$ and $p_{1}$ are subwords of $\varphi(a)$ and $\left.\varphi(b)\right)$.

Thus we can take $L_{\omega}=l^{2}+1$. In what follows we assume that $L_{\omega}=l^{2}+1$. Let $n \geq L_{\omega}$. Then for $n$ there exists a unique pair of numbers $k(n)$ and $s(n)$ such that $s(n)>0, k(n) \in\left\{l, \ldots, l^{2}-1\right\}$ and $k(n) l^{s(n)}<n \leq(k(n)+1) l^{s(n)}$. The number $n-k(n) l^{s(n)}$ is denoted by $r(n)$. We note that $n=k(n) l^{s(n)}+r(n)$ and $r(n) \in\left[1 ; l^{s(n)}\right]$.

For an arbitrary subword $u$ of the word $\omega$ we define the set $N_{u}$ of all pairs of conjugate permutations, and $M_{u}$ as the set of all remaining permutations generated by $u$ as subword of $\omega$. The set of all permutations generated by $u$ is denoted by $H_{u}$. We note that $\left|H_{u}\right|=f(u)$. The cardinality of the set $M_{u}$ is denoted by $m_{u}$, and the cardinality of the set $N_{u}$ is denoted by $n_{u}$. So we have $f(u)=m_{u}+2 n_{u}$.

Let $\mu(n)=\sum_{|u|=n}\left(m_{u}+n_{u}\right)$ and $\chi(n)=\sum_{|u|=n} f(u)$. The main theorem of this section follows:

Theorem 1 Let $n \geq L_{\omega}$ and $\varphi \in Q_{l}$. Then

$$
\sum_{|u|=n} f(u)=(r(n)-1) \mu(k(n)+2)+\left(l^{s(n)}-r(n)+1\right) \chi(k(n)+1) .
$$

Let $u$ be a subword of the word $\omega$ of length $n \geq L_{\omega}$, and $u^{\prime}$ be the ancestor of $u$. Let $I_{u}$ be the set of integer such that for each $\pi \in H_{u}$ there exists a unique integer $m \in I_{u}$ such that $\pi=\pi(u, m)$. Now we define two important maps which will be used in what follows.

For each permutation $\pi$ of $H_{u}$, consider the occurrence $(u, m)$ such that $\pi=\pi(u, m)$ and $m \in I_{u}(m$ is uniquely defined due to the definition of $I_{u}$ ). Then we can define a map $\Psi_{u}: H_{u} \rightarrow H_{u^{\prime}}$ by the rule $\Psi_{u}(\pi)=\pi^{\prime}$, where $\left(u^{\prime}, m^{\prime}\right)$ is the ancestor of the occurrence $(u, m)$ and $\pi^{\prime}=\pi\left(u^{\prime}, m^{\prime}\right)$.

For each permutation $\pi^{\prime}$ of $H_{u^{\prime}}$, consider the occurrence $\left(u^{\prime}, m^{\prime}\right)$ such that $\pi^{\prime}=\pi\left(u^{\prime}, m^{\prime}\right)$ and $m^{\prime} \in I_{u^{\prime}}$ ( $m^{\prime}$ is uniquely defined due to the definition of $I_{u^{\prime}}$ ). Then we can define a map $\Lambda_{u}: H_{u^{\prime}} \rightarrow H_{u}$ by the rule $\Lambda_{u}\left(\pi^{\prime}\right)=\pi$, where $\left(u^{\prime}, m^{\prime}\right)$ is the ancestor of the occurrence $(u, m)$ and $\pi=\pi(u, m)$.

Lemma 8 Let $u$ be a word of length $|u|=x l+r \geq L_{\omega}$ for $1 \leq r \leq l$, and $u^{\prime}$ be the ancestor of $u$ of length $x+2$. Then $f(u)=m_{u^{\prime}}+n_{u^{\prime}}$. 
Proof: Let us prove that $m_{u^{\prime}}+n_{u^{\prime}} \leq\left|H_{u}\right|$. For any word $v$ we define the set $P_{v}$ which contains all permutations of $M_{v}$ and one permutation of each pair of $N_{v}$. It is clear that $\left|P_{v}\right|=m_{v}+n_{v}$. Consider the restriction of the map $\Lambda_{u}$ to the set $P_{u^{\prime}}$. Let $\pi_{1}^{\prime}=\pi\left(u^{\prime}, m_{1}^{\prime}\right)$ and $\pi_{2}^{\prime}=\pi\left(u^{\prime}, m_{2}^{\prime}\right)$ be two different elements of $P_{u^{\prime}}$. Then $\pi_{1}^{\prime} \nsim \pi_{2}^{\prime}$, since otherwise $\left(\pi_{1}^{\prime}, \pi_{2}^{\prime}\right) \in N_{u^{\prime}}$ and it contradicts with the definition of $P_{u^{\prime}}$. Hence Case 3 of Lemma 7 implies that $\Lambda_{u}\left(\pi_{1}^{\prime}\right) \neq \Lambda_{u}\left(\pi_{2}^{\prime}\right)$. Hence $\Lambda_{u}$ is an injective map from $P_{u^{\prime}}$ to $H_{u}$ and $m_{u^{\prime}}+n_{u^{\prime}}=\left|P_{u^{\prime}}\right| \leq\left|H_{u}\right|$.

Let us prove that $\left|H_{u}\right| \leq m_{u^{\prime}}+n_{u^{\prime}}$. Consider the map $\Psi_{u}: H_{u} \rightarrow H_{u^{\prime}}$ defined above. Let $\pi_{1}=$ $\pi\left(u, m_{1}\right)$ and $\pi_{2}=\pi\left(u, m_{2}\right)$ be two different elements of $H_{u}$. Then $\Psi_{u}\left(\pi_{1}\right) \neq \Psi_{u}\left(\pi_{2}\right)$ due to Case 1 of Lemma 7 Since $(x+1) l+1>x l+r$, we have that $|u|<\left(\left|u^{\prime}\right|-1\right) l+1$. Then $\Psi_{u}\left(\pi_{1}\right) \nsim \Psi_{u}\left(\pi_{2}\right)$, since otherwise Case 5 of Lemma 7 would imply that $\pi_{1}=\pi_{2}$. Thus we have proved that $\Psi_{u}$ is an injective map and the image $\Psi_{u}\left[H_{u}\right]$ of $H_{u}$ does not contain conjugate permutations. Since the cardinality of any subset of $H_{u^{\prime}}$ which does not contain conjugate permutations is at most $m_{u^{\prime}}+n_{u^{\prime}}$, we obtain that $\left|H_{u}\right| \leq m_{u^{\prime}}+n_{u^{\prime}}$.

Combining the inequalities proven above, we obtain that

$$
f(u)=\left|H_{u}\right|=m_{u^{\prime}}+n_{u^{\prime}}
$$

The lemma is proved.

Lemma 9 Let $u$ be a word with $|u|=x l+r \geq L_{\omega}$, $u^{\prime}$ be the ancestor of $u$ and $\left|u^{\prime}\right|=x+1$. Then the following statements are true:

1. $f(u)=f\left(u^{\prime}\right)$.

2. If $r=1$, then $m_{u}+n_{u}=m_{u^{\prime}}+n_{u^{\prime}}$.

Proof: 1. Consider the map $\Psi_{u}: H_{u} \rightarrow H_{u^{\prime}}$ defined above. Let $\pi_{1}=\pi\left(u, m_{1}\right)$ and $\pi_{2}=\pi\left(u, m_{2}\right)$ be two different elements of $H_{u}$. Then $\Psi_{u}\left(\pi_{1}\right) \neq \Psi_{u}\left(\pi_{2}\right)$ due to Case 1 of Lemma 7 . Hence $\Psi_{u}$ is an injective map and

$$
f(u)=\left|H_{u}\right| \leq\left|H_{u^{\prime}}\right|=f\left(u^{\prime}\right) .
$$

Let us prove that $f(u) \geq f\left(u^{\prime}\right)$. Consider the map $\Lambda_{u}: H_{u^{\prime}} \rightarrow H_{u}$ defined above. Let $\pi_{1}^{\prime}=\pi\left(u^{\prime}, m_{1}^{\prime}\right)$ and $\pi_{2}^{\prime}=\pi\left(u^{\prime}, m_{2}^{\prime}\right)$ be two different elements of $H_{u^{\prime}}$. Since $x l+1 \leq x l+r$, we have that $|u| \geq$ $\left(\left|u^{\prime}\right|-1\right) l+1$. If $\pi_{1}^{\prime} \nsim \pi_{2}^{\prime}$, then Case 3 of Lemma 7 implies that $\Lambda_{u}\left(\pi_{1}^{\prime}\right) \neq \Lambda_{u}\left(\pi_{2}^{\prime}\right)$. If $\pi_{1}^{\prime} \sim \pi_{2}^{\prime}$, then Cases 4 and 6 of Lemma 7 imply that $\Lambda_{u}\left(\pi_{1}^{\prime}\right) \neq \Lambda_{u}\left(\pi_{2}^{\prime}\right)$. Hence $\Lambda_{u}$ is an injective map and

$$
f\left(u^{\prime}\right)=\left|H_{u^{\prime}}\right| \leq\left|H_{u}\right|=f(u)
$$

So $f(u)=f\left(u^{\prime}\right)$.

2. Since $r=1$, we have $|u|=\left(\left|u^{\prime}\right|-1\right) l+1$. Let us prove that $n_{u} \leq n_{u^{\prime}}$. Consider a pair $\left(\pi_{1}, \pi_{2}\right) \in N_{u}$. Then the pair $\left(\Psi_{u}\left(\pi_{1}\right), \Psi_{u}\left(\pi_{2}\right)\right) \in N_{u^{\prime}}$ due to Case 2 of Lemma 7 . Moreover, two different pairs $\left(\pi_{1}, \pi_{2}\right) \in N_{u}$ and $\left(\pi_{3}, \pi_{4}\right) \in N_{u}$ correspond to two different pairs $\left(\Psi_{u}\left(\pi_{1}\right), \Psi_{u}\left(\pi_{2}\right)\right)$ and $\left(\Psi_{u}\left(\pi_{3}\right), \Psi_{u}\left(\pi_{4}\right)\right)$ due to Case 1 of Lemma 7. Hence $n_{u} \leq n_{u^{\prime}}$.

Let us prove that $n_{u} \geq n_{u^{\prime}}$. Consider a pair $\left(\pi_{1}^{\prime}, \pi_{2}^{\prime}\right) \in N_{u^{\prime}}$. Then the pair $\left(\Lambda_{u}\left(\pi_{1}^{\prime}\right), \Lambda_{u}\left(\pi_{2}^{\prime}\right)\right) \in N_{u}$ due to Case 4 of Lemma 7. Moreover, two different pairs $\left(\pi_{1}^{\prime}, \pi_{2}^{\prime}\right) \in N_{u^{\prime}}$ and $\left(\pi_{3}^{\prime}, \pi_{4}^{\prime}\right) \in N_{u^{\prime}}$ correspond to two different pairs $\left(\Lambda_{u}\left(\pi_{1}^{\prime}\right), \Lambda_{u}\left(\pi_{2}^{\prime}\right)\right)$ and $\left(\Lambda_{u}\left(\pi_{3}^{\prime}\right), \Lambda_{u}\left(\pi_{4}^{\prime}\right)\right)$ due to Case 3 of Lemma 7 Hence $n_{u} \geq n_{u^{\prime}}$. 
Combining the inequalities proven above, we obtain that $n_{u}=n_{u^{\prime}}$. Moreover $f(u)=f\left(u^{\prime}\right)$ due to Case 1 of this Lemma. So

$$
m_{u}+n_{u}=f(u)-n_{u}=f\left(u^{\prime}\right)-n_{u^{\prime}}=m_{u^{\prime}}+n_{u^{\prime}} .
$$

Recall that $\varphi_{i ; j}(v)$ is the word obtained from $\varphi(v)$ by erasing $i$ symbols to the left and $j$ symbols to the right where $0 \leq i<l$ and $0 \leq j<l$. The set of all subwords of $\omega$ of length $n$ is denoted by $F_{\omega}(n)$.

Lemma 10 Let $n=x l+r \geq L_{\omega}$, where $1 \leq r \leq l$. Then the following statements are true:

1. $\chi(n)=(r-1) \mu(x+2)+(l-r+1) \chi(x+1)$.

2. $\mu(n)=\chi(n)$ for $r>1$ and $\mu(x l+1)=l \mu(x+1)$.

Proof: Since $n \geq L_{\omega}$, each word $u \in F_{\omega}(n)$ has a unique interpretation $\langle v, i, j\rangle$, where $0 \leq i<l$, $0 \leq j<l$ and $v$ is the ancestor of $u$. By the definition of interpretation we have $|u|=|v| l-i-j$. Hence $i+j \equiv l-r(\bmod l)$. So $|v|=x+2$ with $i+j=2 l-r$ or $|v|=x+1$ with $i+j=l-r$.

Let

$$
A_{1}=\left\{\varphi_{i ; j}(v) \mid v \in F_{\omega}(x+2), i+j=2 l-r\right\}
$$

and

$$
A_{2}=\left\{\varphi_{i ; j}(v) \mid v \in F_{\omega}(x+1), i+j=l-r\right\} .
$$

Let us prove the following assertion:

Proposition 4 1. $F_{\omega}(n)=A_{1} \cup A_{2}$ and $A_{1} \bigcap A_{2}=\emptyset$.

2. $F_{\omega}(n)=A_{2}$ for $r=1$.

Proof: Let us prove that $A_{1} \bigcap A_{2}=\emptyset$. Let $u \in A_{1} \bigcap A_{2}$. Then $u$ has interpretations with $|v|=x+2$ and $|v|=x+1$. So $u$ has two different interpretations. It contradicts with $|u| \geq L_{\omega}$. So $A_{1} \bigcap A_{2}=\emptyset$.

Let us prove that $A_{1} \bigcup A_{2} \subseteq F_{\omega}(n)$. Let $v$ be a subword of $\omega$. Since $\omega$ is a fixed point of morphism $\varphi$, $\varphi(v)$ is a subword of $\omega$. By the definition of $\varphi_{i ; j}(v)$ we have that $\varphi_{i ; j}(v)$ is a subword of $\varphi(v)$. So $\varphi_{i ; j}(v)$ is a subword of $\omega$. Let $u \in A_{1}$. Then $u=\varphi_{i ; j}(v)$ for some subword $v$ of length $x+2$ and $i+j=2 l-r$. Then

$$
|u|=|v| l-i-j=(x+2) l-2 l+r=x l+r .
$$

Since $\varphi_{i ; j}(v)$ is a subword of $\omega$ and $|u|=x l+r=n$, we obtain that $u \in F_{\omega}(n)$. Let $u \in A_{2}$. Then $u=\varphi_{i ; j}(v)$ for some subword $v$ of length $x+1$ and $i+j=l-r$. Then

$$
|u|=|v| l-i-j=(x+1) l-l+r=x l+r .
$$

Since $\varphi_{i ; j}(v)$ is a subword of $\omega$ and $|u|=x l+r=n$, we obtain that $u \in F_{\omega}(n)$. Thus $A_{1} \bigcup A_{2} \subseteq F_{\omega}(n)$.

Let us prove that $F_{\omega}(n) \subseteq A_{1} \bigcup A_{2}$. Let $u \in F_{\omega}(n)$. Then $u$ has unique interpretation $\langle v, i, j\rangle$, where $0 \leq i<l, 0 \leq j<l$ and $v$ is the ancestor of $u$. Then $u=\varphi_{i ; j}(v)$ due to the definition of $\varphi_{i ; j}(v)$ and as we proved above $|v|=x+2$ or $|v|=x+1$. Hence $u \in A_{1}$ or $u \in A_{2}$. Thus $F_{\omega}(n) \subseteq A_{1} \bigcup A_{2}$ and $F_{\omega}(n)=A_{1} \bigcup A_{2}$. 
It remains to consider the case when $r=1$. Since $0 \leq i<l$ and $0 \leq j<l$, we have $i+j<2 l-1$. Then $A_{1}=\emptyset$ due to the definition of $A_{1}$. So $F_{\omega}(n)=A_{2}$ for $r=1$ due to Case 1 of this Proposition.

\section{Proof of Lemma 10:}

1. We have $F_{\omega}(n)=A_{1} \bigcup A_{2}$ due to Proposition 4 . Then

$$
\chi(n)=\sum_{|u|=n} f(u)=\sum_{u \in A_{1}} f(u)+\sum_{u \in A_{2}} f(u) .
$$

Let $u=\varphi_{i ; j}(v) \in A_{1}$. Then $v$ is the ancestor of $u,|u|=n=x l+r$ and $|v|=x+2$. Then Lemma 8 implies that $f(u)=m_{v}+n_{v}$. So

$\sum_{u \in A_{1}} f(u)=\sum_{|v|=x+2, i+j=2 l-r} f\left(\varphi_{i ; j}(v)\right)=\sum_{|v|=x+2}(r-1)\left(m_{v}+n_{v}\right)=(r-1) \sum_{|v|=x+2}\left(m_{v}+n_{v}\right)=(r-1) \mu(x+2)$.

If $u=\varphi_{i ; j}(v) \in A_{2}$, then $v$ is the ancestor of $u,|u|=n=x l+r$ and $|v|=x+1$. Then Lemma 9 implies that $f(u)=f(v)$. So

$\sum_{u \in A_{2}} f(u)=\sum_{|v|=x+1, i+j=l-r} f\left(\varphi_{i ; j}(v)\right)=\sum_{|v|=x+1}(l-r+1) f(v)=(l-r+1) \sum_{|v|=x+1} f(v)=(l-r+1) \chi(x+1)$.

Thus $\chi(n)=(r-1) \mu(x+2)+(l-r+1) \chi(x+1)$.

2. Let $r>1$. Consider an arbitrary word $u$ of length $n$. Let $n_{u}>0$. Then due to Case 2 of Lemma 7 , the existence of two conjugate permutations $\pi\left(u, m_{1}\right)$ and $\pi\left(u, m_{2}\right)$ would imply that $|u|=\left(\left|u^{\prime}\right|-1\right) l+1$, so that $n_{u}=0$. Thus

$$
\mu(n)=\sum_{|u|=n}\left(m_{u}+n_{u}\right)=\sum_{|u|=n} f(u)=\chi(n)
$$

for $r>1$.

Consider the case when $r=1$. We have $F_{\omega}(n)=A_{2}$ by Proposition 4 Then

$$
\mu(n)=\sum_{|u|=n}\left(m_{u}+n_{u}\right)=\sum_{u \in A_{2}}\left(m_{u}+n_{u}\right) .
$$

Let $u=\varphi_{i ; j}(v) \in A_{2}$. Then $v$ is the ancestor of $u,|u|=n=x l+r$ and $|v|=x+1$. Then Lemma 9 implies that $m_{u}+n_{u}=m_{v}+n_{v}$. So

$$
\sum_{u \in A_{2}}\left(m_{u}+n_{u}\right)=\sum_{|v|=x+1, i+j=l-1} f\left(\varphi_{i ; j}(v)\right)=\sum_{|v|=x+1} l\left(m_{v}+n_{v}\right)=l \sum_{|v|=x+1}\left(m_{v}+n_{v}\right)=l \mu(x+1) .
$$

Lemma 11 Let $n \geq L_{\omega}$. Then $\mu(n)=\chi(n)$ for $r(n)>1$ and $\mu(n)=\mu(k(n)+1) l^{s(n)}$ for $r(n)=1$.

Proof: Let us prove by the induction on $s(n)$ that $\mu(n)=\chi(n)$ for $r(n)>1$. For $s(n)=1$ it is true due to Case 2 of Lemma 10 Let us prove the induction step. Let $n=x l+r_{0}$ for $r_{0} \in[1, l]$. If $r_{0}>1$, then 
$\mu(n)=\chi(n)$ due to Case 2 of Lemma 10 Consider the case when $r_{0}=1$. Then $n=x l+1$ and we have $\mu(x l+1)=l \mu(x+1)$ due to Case 2 of Lemma 10 . Since

$$
k(n) l^{s(n)}+2 \leq n \leq(k(n)+1) l^{s(n)},
$$

we have

$$
k(n) l^{s(n)-1}+\frac{1}{l} \leq x \leq(k(n)+1) l^{s(n)-1}-\frac{1}{l} .
$$

Hence

$$
k(n) l^{s(n)-1}+1 \leq x \leq(k(n)+1) l^{s(n)-1}-1 .
$$

So $x+1=k(n) l^{s(n)-1}+r^{\prime}$ and $r^{\prime} \in\left[2, l^{s(n)-1}\right]$. Then by the induction assumption we have that $\mu(x+1)=\chi(x+1)$. Hence $\mu(x l+1)=l \chi(x+1)$. Since $\chi(x l+1)=l \chi(x+1)$ due to Case 1 of Lemma 10, we obtain that $\mu(x l+1)=\chi(x l+1)$.

Let us prove by the induction on $s(n)$ that

$$
\mu(n)=\mu(k(n)+1) l^{s(n)}
$$

for $r(n)=1$. For $s(n)=1$ it is true due to Case 2 of Lemma 10 Let us prove the induction step. We have $n=x l+1$ for some $x$. Then $\mu(x l+1)=l \mu(x+1)$ due to Case 2 of Lemma 10. Moreover $x+1=k(n) l^{s(n)-1}+1$. Then by the induction assumption we have that

$$
\mu(x+1)=\mu(k(n)+1) l^{s(n)-1} .
$$

Hence

$$
\mu(n)=\mu(k(n)+1) l^{s(n)} .
$$

\section{Proof of Theorem 1;}

Let us prove Theorem 1 by the induction on $s(n)$. For $s(n)=1$ it is true due to Case 1 of Lemma 10 Let us prove the induction step. Let $n=x l+r_{0}$ for $r_{0} \in[1, l]$. Since

$$
k(n) l^{s(n)}+1 \leq n \leq(k(n)+1) l^{s(n)},
$$

we have

$$
k(n) l^{s(n)-1}+\frac{1-l}{l} \leq x \leq(k(n)+1) l^{s(n)-1}-\frac{1}{l} .
$$

Hence

$$
k(n) l^{s(n)-1} \leq x \leq(k(n)+1) l^{s(n)-1}-1 .
$$

Hence $x+1 \in\left[k(n) l^{s(n)-1}+1,(k(n)+1) l^{s(n)-1}\right]$ and $x+2 \in\left[k(n) l^{s(n)-1}+2,(k(n)+1) l^{s(n)-1}+1\right]$. If $x+2 \in\left[k(n) l^{s(n)-1}+2,(k(n)+1) l^{s(n)-1}\right]$, then $\mu(x+2)=\chi(x+2)$ due to Lemma 11 . By Lemma 10 we have

$$
\chi(n)=\left(r_{0}-1\right) \mu(x+2)+\left(l-r_{0}+1\right) \chi(x+1) .
$$

Since $\mu(x+2)=\chi(x+2)$, we have that

$$
\chi(n)=\left(r_{0}-1\right) \chi(x+2)+\left(l-r_{0}+1\right) \chi(x+1) .
$$


By the induction assumption we have

$$
\chi(x+2)=\left(x+1-k(n) l^{s(n)-1}\right) \mu(k(n)+2)+\left((k(n)+1) l^{s(n)-1}-x-1\right) \chi(k(n)+1)
$$

and

$$
\chi(x+1)=\left(x-k(n) l^{s(n)-1}\right) \mu(k(n)+2)+\left((k(n)+1) l^{s(n)-1}-x\right) \chi(k(n)+1) .
$$

So $\chi(n)=\left(r_{0}-1\right) \chi(x+2)+\left(l-r_{0}+1\right) \chi(x+1)=\left(x l+r_{0}-k(n) l^{s(n)}-1\right) \mu(k(n)+2)+((k(n)+$ 1) $\left.l^{s(n)}-x l-r_{0}+1\right) \chi(k(n)+1)=(r(n)-1) \mu(k+2)+\left(l^{s(n)}-r(n)+1\right) \chi(k+1)$.

It remains to consider the case when $x+2=(k(n)+1) l^{s(n)-1}+1$. Then we have

$$
\mu(x+2)=\mu(k(n)+2) l^{s(n)-1}
$$

due to Lemma 11 By the induction assumption we have

$$
\chi(x+1)=\left(x-k(n) l^{s(n)-1}\right) \mu(k(n)+2)+\left((k(n)+1) l^{s(n)-1}-x\right) \chi(k(n)+1) .
$$

Since $x=(k(n)+1) l^{s(n)-1}-1$, we have

$$
\chi(x+1)=\left(l^{s(n)-1}-1\right) \mu(k(n)+2)+\chi(k(n)+1) .
$$

So

$\chi(n)=\left(r_{0}-1\right) \mu(x+2)+\left(l-r_{0}+1\right) \chi(x+1)=\left(l^{s(n)}-l+r_{0}-1\right) \mu(k(n)+2)+\left(l-r_{0}+1\right) \chi(k(n)+1)$.

Since $r(n)=n-k(n) l^{s(n)}=x l+r_{0}-k(n) l^{s(n)}=l^{s(n)}-l+r_{0}$, we have

$$
\chi(n)=(r(n)-1) \mu((n) k+2)+\left(l^{s(n)}-r(n)+1\right) \chi(k(n)+1) .
$$

\section{Special words}

Recall that a subword $v$ of the word $\omega$ is called special (in $\omega$ ) if $v 0$ and $v 1$ are also subwords of $\omega$. The set of all the special words of length $n$ is denoted by $B(n)$. Note that the unique interpretation of any special word $v$ of length at least $L_{\omega}$ is equal to $\left\langle v^{\prime}, i, 0\right\rangle$. Indeed, if $j>0$, then $v$ is uniquely completed to the right to a full block, and thus only one of the words $v 0$ and $v 1$ is a subword of $\omega$.

Consider a special word $v$. Let $a$ be the first letter of $v$ and $b=\{0,1\} \backslash a$. Then $v b$ cannot generate conjugate permutations. Let $G_{v}=H_{v a} \cap H_{v b}$. The cardinality of $G_{v}$ is denoted by $g(v)$. So $g(v)$ is the number of common permutations generated by words $v 0$ and $v 1$. Let $K_{v}$ be the set and $k_{v}$ be the number of permutations of $M_{v a}$ which also belong to $H_{v b}$. Let $T_{v}$ be the set and $t_{v}$ be the number of permutations of $M_{v a}$ each of which is conjugate to some permutation of $H_{v b}$. Let $R_{v}$ be the set and $r_{v}$ be the number of permutations of $H_{v a} \backslash M_{v a}$ which also belong to $H_{v b}$. Thus if $\left(\pi_{1}, \pi_{2}\right) \in N_{v a}$ and $\pi_{1} \in R_{v}$, then $\pi_{2}$ is conjugate to some permutation of $H_{v b}$.

Let $\alpha(n)=\sum_{z \in B(n)} g(z)$ and $\beta(n)=\sum_{z \in B(n)}\left(k_{z}+t_{z}+r_{z}\right)$.

The main theorem of this section is: 
Theorem 2 Let $n \geq L_{\omega}$ and $\varphi \in Q_{l}$. Then $\sum_{v \in B(n)} g(v)=\beta(k(n)+1)$ for $r(n)<l^{s(n)}$ and $\sum_{v \in B(n)} g(v)=\alpha(k(n)+1)$ for $r(n)=l^{s(n)}$.

To prove Theorem 2, we prove several auxiliary assertions. Consider the following Lemma (Lemma 1 from Makarov (2006)).

Lemma 12 Let $u=u_{1} \ldots u_{n}$ and $v=v_{1} \ldots v_{n}$ be two subwords of any infinite binary word such that $u_{i} \neq v_{i}$ for some $1 \leq i \leq n-1$. Then $u$ and $v$ do not generate equal permutations.

So, two words can generate equal permutations only if they are $v 0$ and $v 1$ for some special word $v$. Since the sum $\sum_{|u|=n} f(u)$ can be calculated as it is shown above, it remains to consider what happens with words $v 0$ and $v 1$ for all special words $v$.

Lemma 13 Let $\left(v 0, m_{1}\right)$ and $\left(v 1, m_{2}\right)$ be some occurrences of words $v 0$ and $v 1$ with $|v|=n \geq L_{\omega}$, $\left(v^{\prime} 0, m_{1}^{\prime}\right)$ and $\left(v^{\prime} 1, m_{2}^{\prime}\right)$ be the ancestors of occurrences of $\left(v 0, m_{1}\right)$ and $\left(v 1, m_{2}\right)$ with $\left|v^{\prime}\right|=k$, where $\pi\left(v^{\prime} 0, m_{1}^{\prime}\right)=\pi\left(v^{\prime} 1, m_{2}^{\prime}\right)$. Then $\pi\left(v 0, m_{1}\right)=\pi\left(v 1, m_{2}\right)$.

Proof: It is clear that $\left(v^{\prime}, m_{1}^{\prime}\right)$ and $\left(v^{\prime}, m_{2}^{\prime}\right)$ are the ancestors of occurrences $\left(v, m_{1}\right)$ and $\left(v, m_{2}\right)$. Note that $\pi\left(v, m_{1}\right)=\pi\left(v, m_{2}\right)$ since otherwise $\pi\left(v^{\prime}, m_{1}^{\prime}\right) \neq \pi\left(v^{\prime}, m_{2}^{\prime}\right)$ due to Case 1 of Lemma 7 and $\pi\left(v^{\prime} 0, m_{1}^{\prime}\right) \neq \pi\left(v^{\prime} 1, m_{2}^{\prime}\right)$.

Consider $1 \leq t<n+1$ and the relations between the element number $t$ and the last element of permutations $\pi\left(v 0, m_{1}\right)$ and $\pi\left(v 1, m_{2}\right)$. Lemma 5 implies that inequality

$$
\gamma\left(R_{\omega}\left(m_{1}+t\right), R_{\omega}\left(m_{1}+n+1\right)\right) \neq \gamma\left(R_{\omega}\left(m_{2}+t\right), R_{\omega}\left(m_{2}+n+1\right)\right)
$$

may hold only if

$$
\gamma\left(R_{\omega}\left(m_{1}^{\prime}+t^{\prime}\right), R_{\omega}\left(m_{1}^{\prime}+k+1\right)\right) \neq \gamma\left(R_{\omega}\left(m_{2}^{\prime}+t^{\prime}\right), R_{\omega}\left(m_{2}^{\prime}+k+1\right)\right)
$$

which is impossible since $\pi\left(v^{\prime} 0, m_{1}^{\prime}\right)=\pi\left(v^{\prime} 1, m_{2}^{\prime}\right)$. Hence

$$
\gamma\left(R_{\omega}\left(m_{1}+t\right), R_{\omega}\left(m_{1}+n+1\right)\right)=\gamma\left(R_{\omega}\left(m_{2}+t\right), R_{\omega}\left(m_{2}+n+1\right)\right)
$$

for any $1 \leq t<n+1$. Thus we have proved that $\pi\left(v 0, m_{1}\right)=\pi\left(v 1, m_{2}\right)$.

Lemma 14 Let $\left(v 0, m_{1}\right)$ and $\left(v 1, m_{2}\right)$ be some occurrences of words $v 0$ and $v 1$ with $|v|=n \geq L_{\omega}$, $\left(v^{\prime} 0, m_{1}^{\prime}\right)$ and $\left(v^{\prime} 1, m_{2}^{\prime}\right)$ be the ancestors of $\left(v 0, m_{1}\right)$ and $\left(v 1, m_{2}\right)$ with $\left|v^{\prime}\right|=k$ where $\pi\left(v^{\prime} 0, m_{1}^{\prime}\right) \neq$ $\pi\left(v^{\prime} 1, m_{2}^{\prime}\right)$ and $\pi\left(v^{\prime} 0, m_{1}^{\prime}\right) \nsim \pi\left(v^{\prime} 1, m_{2}^{\prime}\right)$. Then $\pi\left(v 0, m_{1}\right) \neq \pi\left(v 1, m_{2}\right)$ and $\pi\left(v 0, m_{1}\right) \nsim \pi\left(v 1, m_{2}\right)$.

Proof: Since $v$ is a special word, we obtain that words $v 0$ and $v 1$ have the interpretations $\left\langle v^{\prime} 0, i, l-1\right\rangle$ and $\left\langle v^{\prime} 1, i, l-1\right\rangle$, where $0 \leq i<l$. Since $\pi\left(v^{\prime} 0, m_{1}^{\prime}\right) \neq \pi\left(v^{\prime} 1, m_{2}^{\prime}\right)$ and $\pi\left(v^{\prime} 0, m_{1}^{\prime}\right) \nsim \pi\left(v^{\prime} 1, m_{2}^{\prime}\right)$, we obtain that two cases are possible.

In the first case

$$
\gamma\left(R_{\omega}\left(m_{1}^{\prime}+t^{\prime}\right), R_{\omega}\left(m_{1}^{\prime}+s^{\prime}\right)\right) \neq \gamma\left(R_{\omega}\left(m_{2}^{\prime}+t^{\prime}\right), R_{\omega}\left(m_{2}^{\prime}+s^{\prime}\right)\right)
$$

for some $1 \leq t^{\prime}<s^{\prime}<k+1$. Then $\pi\left(v^{\prime}, m_{1}^{\prime}\right) \neq \pi\left(v^{\prime}, m_{2}^{\prime}\right)$. If $\pi\left(v^{\prime}, m_{1}^{\prime}\right) \nsim \pi\left(v^{\prime}, m_{2}^{\prime}\right)$, then $\pi\left(v, m_{1}\right) \neq$ $\pi\left(v, m_{2}\right)$ due to Case 3 of Lemma 7. If $\pi\left(v^{\prime}, m_{1}^{\prime}\right) \sim \pi\left(v^{\prime}, m_{2}^{\prime}\right)$, then $\pi\left(v, m_{1}\right) \neq \pi\left(v, m_{2}\right)$ due to Case 4 or 6 of Lemma 7 since $|v| \geq\left(\left|v^{\prime}\right|-1\right) l+1$. Hence $\pi\left(v 0, m_{1}\right) \neq \pi\left(v 1, m_{2}\right)$ and $\pi\left(v 0, m_{1}\right) \nsim \pi\left(v 1, m_{2}\right)$. 
In the second case

$$
\gamma\left(R_{\omega}\left(m_{1}^{\prime}+t^{\prime}\right), R_{\omega}\left(m_{1}^{\prime}+k+1\right)\right) \neq \gamma\left(R_{\omega}\left(m_{2}^{\prime}+t^{\prime}\right), R_{\omega}\left(m_{2}^{\prime}+k+1\right)\right)
$$

for some $1<t^{\prime}<k+1$. Then Lemma 5 implies that

$$
\gamma\left(R_{\omega}\left(\left(m_{1}^{\prime}+t^{\prime}-1\right) l+1\right), R_{\omega}\left(\left(m_{1}^{\prime}+k\right) l+1\right)\right) \neq \gamma\left(R_{\omega}\left(\left(m_{2}^{\prime}+t^{\prime}-1\right) l+1\right), R_{\omega}\left(\left(m_{2}^{\prime}+k\right) l+1\right)\right) .
$$

Since $t^{\prime}>1$, we obtain that the symbols $\omega_{\left(m_{1}^{\prime}+t^{\prime}-1\right) l+1}$ and $\omega_{\left(m_{2}^{\prime}+t^{\prime}-1\right) l+1}$ are not the first symbols of words $\omega_{m_{1}+1} \ldots \omega_{m_{1}+n+1}$ and $\omega_{m_{2}+1} \ldots \omega_{m_{2}+n+1}$. Thus we have proved that $\pi\left(v 0, m_{1}\right) \neq \pi\left(v 1, m_{2}\right)$ and $\pi\left(v 0, m_{1}\right) \nsim \pi\left(v 1, m_{2}\right)$.

Lemma 15 Let $\left(v 0, m_{1}\right)$ and $\left(v 1, m_{2}\right)$ be some occurrences of words $v 0$ and $v 1$ with $|v|=n \geq L_{\omega}$, $\left(v^{\prime} 0, m_{1}^{\prime}\right)$ and $\left(v^{\prime} 1, m_{2}^{\prime}\right)$ be the ancestors of $\left(v 0, m_{1}\right)$ and $\left(v 1, m_{2}\right)$ with $\left|v^{\prime}\right|=k$. Then the following statements are true:

1. If $\pi\left(v^{\prime} 0, m_{1}^{\prime}\right) \sim \pi\left(v^{\prime} 1, m_{2}^{\prime}\right)$ and $|v|=l\left|v^{\prime}\right|$, then $\pi\left(v 0, m_{1}\right) \sim \pi\left(v 1, m_{2}\right)$.

2. If $\pi\left(v 0, m_{1}\right) \sim \pi\left(v 1, m_{2}\right)$, then $\pi\left(v^{\prime} 0, m_{1}^{\prime}\right) \sim \pi\left(v^{\prime} 1, m_{2}^{\prime}\right)$ and $|v|=l\left|v^{\prime}\right|$.

3. If $\pi\left(v^{\prime} 0, m_{1}^{\prime}\right) \sim \pi\left(v^{\prime} 1, m_{2}^{\prime}\right)$ and $|v|<l\left|v^{\prime}\right|$, then $\pi\left(v 0, m_{1}\right)=\pi\left(v 1, m_{2}\right)$.

Proof: Since $v$ is a special word, we obtain that words $v 0$ and $v 1$ have the interpretations $\left\langle v^{\prime} 0, i, l-1\right\rangle$ and $\left\langle v^{\prime} 1, i, l-1\right\rangle$, where $0 \leq i<l$.

First let us prove the first and third statements. Since $\pi\left(v^{\prime} 0, m_{1}^{\prime}\right) \sim \pi\left(v^{\prime} 1, m_{2}^{\prime}\right)$, by the definition of $\sim$ we have that $\pi\left(v^{\prime}, m_{1}^{\prime}\right)=\pi\left(v^{\prime}, m_{2}^{\prime}\right)$. It is clear that $\left(v^{\prime}, m_{1}^{\prime}\right)$ and $\left(v^{\prime}, m_{2}^{\prime}\right)$ are the ancestors of occurrences $\left(v, m_{1}\right)$ and $\left(v, m_{2}\right)$. Note that $\pi\left(v, m_{1}\right)=\pi\left(v, m_{2}\right)$ since otherwise $\pi\left(v^{\prime}, m_{1}^{\prime}\right) \neq \pi\left(v^{\prime}, m_{2}^{\prime}\right)$ due to Case 1 of Lemma7

Consider $1 \leq t<n+1$. Then Lemma 5 implies that either

$$
\gamma\left(R_{\omega}\left(m_{1}+t\right), R_{\omega}\left(m_{1}+n+1\right)\right)=\gamma\left(R_{\omega}\left(m_{2}+t\right), R_{\omega}\left(m_{2}+n+1\right)\right),
$$

or $m_{1}+t=\left(m_{1}^{\prime}+t^{\prime}-1\right) l+1, m_{2}+t=\left(m_{2}^{\prime}+t^{\prime}-1\right) l+1$ for some $t^{\prime}$ and

$$
\gamma\left(R_{\omega}\left(m_{1}^{\prime}+t^{\prime}\right), R_{\omega}\left(m_{1}^{\prime}+k+1\right)\right) \neq \gamma\left(R_{\omega}\left(m_{2}^{\prime}+t^{\prime}\right), R_{\omega}\left(m_{2}^{\prime}+k+1\right)\right) .
$$

Consider the second case. Then since $\pi\left(v^{\prime} 0, m_{1}^{\prime}\right) \sim \pi\left(v^{\prime} 1, m_{2}^{\prime}\right)$, we have

$$
\gamma\left(R_{\omega}\left(m_{1}^{\prime}+t^{\prime}\right), R_{\omega}\left(m_{1}^{\prime}+k+1\right)\right)=\gamma\left(R_{\omega}\left(m_{2}^{\prime}+t^{\prime}\right), R_{\omega}\left(m_{2}^{\prime}+k+1\right)\right)
$$

for $1<t^{\prime}<k+1$.

Consider the case of $t^{\prime}=1$. Then $m_{1}+t=m_{1}^{\prime} l+1$ and $m_{2}+t=m_{2}^{\prime} l+1$. It is possible only if $|v|=l\left|v^{\prime}\right|$. So if $|v|<l\left|v^{\prime}\right|$, then $\pi\left(v 0, m_{1}\right)=\pi\left(v 1, m_{2}\right)$. If $|v|=l\left|v^{\prime}\right|$, then

$$
\gamma\left(R_{\omega}\left(m_{1}+t\right), R_{\omega}\left(m_{1}+n+1\right)\right) \neq \gamma\left(R_{\omega}\left(m_{2}+t\right), R_{\omega}\left(m_{2}+n+1\right)\right)
$$

due to Lemma 5 and $\pi\left(v 0, m_{1}\right) \sim \pi\left(v 1, m_{2}\right)$. 
Now let us prove the second statement. We have $\pi\left(v 0, m_{1}\right) \sim \pi\left(v 1, m_{2}\right)$. Hence we obtain that

$$
\gamma\left(R_{\omega}\left(m_{1}^{\prime} l+i+1\right), R_{\omega}\left(\left(m_{1}^{\prime}+k\right) l+1\right)\right) \neq \gamma\left(R_{\omega}\left(m_{2}^{\prime} l+i+1\right), R_{\omega}\left(\left(m_{2}^{\prime}+k\right) l+1\right)\right) .
$$

Lemma 5 implies that $m_{1}^{\prime} l+i+1 \equiv 1(\bmod l)$ or $m_{2}^{\prime} l+i+1 \equiv 1(\bmod l)$. Then $i=0$ and $n=k l$.

Since $\pi\left(v 0, m_{1}\right) \sim \pi\left(v 1, m_{2}\right)$, we obtain $\pi\left(v, m_{1}\right)=\pi\left(v, m_{2}\right)$. It is clear that $\left(v^{\prime}, m_{1}^{\prime}\right)$ and $\left(v^{\prime}, m_{2}^{\prime}\right)$ are the ancestors of occurrences $\left(v, m_{1}\right)$ and $\left(v, m_{2}\right)$. Since $n=k l$, we have $|v|>\left(\left|v^{\prime}\right|-1\right) l+1$. Then $\pi\left(v^{\prime}, m_{1}^{\prime}\right)=\pi\left(v^{\prime}, m_{2}^{\prime}\right)$ since otherwise either $\pi\left(v, m_{1}\right) \neq \pi\left(v, m_{2}\right)$ due to Case 3 of Lemma 7 , or $\pi\left(v, m_{1}\right) \neq \pi\left(v, m_{2}\right)$ due to Case 6 of Lemma 7. Hence we obtain that

$$
\gamma\left(R_{\omega}\left(m_{1}^{\prime}+t^{\prime}\right), R_{\omega}\left(m_{1}^{\prime}+s^{\prime}\right)\right)=\gamma\left(R_{\omega}\left(m_{2}^{\prime}+t^{\prime}\right), R_{\omega}\left(m_{2}^{\prime}+s^{\prime}\right)\right)
$$

for $1 \leq t^{\prime}<s^{\prime}<k+1$.

It remains to consider the case $s^{\prime}=k+1$. Since $\pi\left(v 0, m_{1}\right) \sim \pi\left(v 1, m_{2}\right)$, we have that $\pi\left(v 0, m_{1}\right)$ and $\pi\left(v 1, m_{2}\right)$ differ only in relations of extreme elements. Then Lemma 5 implies that

$$
\gamma\left(R_{\omega}\left(m_{1}^{\prime}+t^{\prime}\right), R_{\omega}\left(m_{1}^{\prime}+k+1\right)\right)=\gamma\left(R_{\omega}\left(m_{2}^{\prime}+t^{\prime}\right), R_{\omega}\left(m_{2}^{\prime}+k+1\right)\right)
$$

for $2 \leq t^{\prime}<k+1$. In addition,

$$
\gamma\left(R_{\omega}\left(m_{1} l+1\right), R_{\omega}\left(\left(m_{1}+k\right) l+1\right)\right) \neq \gamma\left(R_{\omega}\left(m_{1} l+1\right), R_{\omega}\left(\left(m_{1}+k\right) l+1\right)\right) .
$$

Hence Lemma 5 implies that

$$
\gamma\left(R_{\omega}\left(m_{1}^{\prime}+1\right), R_{\omega}\left(m_{1}^{\prime}+k+1\right)\right) \neq \gamma\left(R_{\omega}\left(m_{2}^{\prime}+t\right), R_{\omega}\left(m_{2}^{\prime}+k+1\right)\right) .
$$

Thus we have proved that $\pi\left(v^{\prime} 0, m_{1}^{\prime}\right) \sim \pi\left(v^{\prime} 1, m_{2}^{\prime}\right)$ and $n=k l$.

Lemma 16 Let $v$ be a special word with the ancestor $v^{\prime}$ with $|v| \geq L_{\omega}$, and $|v|<l\left|v^{\prime}\right|$. Then $g(v)=$ $k_{v^{\prime}}+t_{v^{\prime}}+r_{v^{\prime}}$.

Proof: Due to Case 2 of Lemma 15 the existence of two conjugate permutations $\pi\left(v 0, m_{1}\right)$ and $\pi\left(v 1, m_{2}\right)$ would imply that $|v|=\left|v^{\prime}\right| l$, so we have $t_{v}=r_{v}=0$.

Let us prove that $k_{v} \leq k_{v^{\prime}}+t_{v^{\prime}}+r_{v^{\prime}}$. Let $a$ be the first letter of $v$ and $b=\{0,1\} \backslash a$. For each permutation $\tau$ of $K_{v}$, consider occurrences $\left(v a, m_{1}\right)$ and $\left(v b, m_{2}\right)$ of words $v a$ and $v b$ such that $\tau=$ $\pi\left(v a, m_{1}\right)=\pi\left(v b, m_{2}\right)$. Then either $\pi\left(v^{\prime} a, m_{1}^{\prime}\right)=\pi\left(v^{\prime} b, m_{2}^{\prime}\right)$, or $\pi\left(v^{\prime} a, m_{1}^{\prime}\right) \sim \pi\left(v^{\prime} b, m_{2}^{\prime}\right)$ due to Lemma 14 . If $\pi\left(v^{\prime} a, m_{1}^{\prime}\right)=\pi\left(v^{\prime} b, m_{2}^{\prime}\right)$, then

$$
\Psi_{v a}(\tau)=\tau^{\prime} \in\left(M_{v^{\prime} a} \cup H_{v^{\prime} a} \backslash M_{v^{\prime} a}\right) \cap H_{v^{\prime} b}=K_{v^{\prime}} \cup R_{v^{\prime}} .
$$

If $\pi\left(v^{\prime} a, m_{1}^{\prime}\right) \sim \pi\left(v^{\prime} b, m_{2}^{\prime}\right)$, then either $\Psi_{v a}(\tau)=\tau^{\prime} \in T_{v^{\prime}}$, or $\Psi_{v a}(\tau)=\tau^{\prime} \sim \tau^{\prime \prime}=\pi\left(v^{\prime} a, m_{3}^{\prime}\right)$ and $\tau^{\prime \prime} \in R_{v^{\prime}}$. Let $\tau_{3}=\pi\left(v a, m_{3}\right)$ and $\tau_{4}=\pi\left(v a, m_{4}\right)$ be two different elements of $K_{v}$. Then $\Psi_{v a}\left(\tau_{3}\right) \neq \Psi_{v a}\left(\tau_{4}\right)$ due to Case 1 of Lemma 7 and $\Psi_{v a}\left(\tau_{3}\right) \nsim \Psi_{v a}\left(\tau_{4}\right)$ due to Case 5 of Lemma 7 . Hence $\Psi_{v a}$ is an injective map from $K_{v}$ to $H_{v^{\prime} a}$ and $\Psi_{v a}\left[K_{v}\right]$ does not contain conjugate permutations. Hence

$$
k_{v}=\left|K_{v}\right| \leq k_{v^{\prime}}+t_{v^{\prime}}+r_{v^{\prime}} .
$$


Let us prove that $k_{v^{\prime}}+t_{v^{\prime}}+r_{v^{\prime}} \leq k_{v}$. Let $a$ be the first letter of $v^{\prime}$ and $b=\{0,1\} \backslash a$. For each permutation $\tau^{\prime}$ of $K_{v^{\prime}} \cup T_{v^{\prime}} \cup R_{v^{\prime}}$ consider arbitrary occurrences $\left(v^{\prime} a, m_{1}^{\prime}\right)$ and $\left(v^{\prime} b, m_{2}^{\prime}\right)$ of words $v^{\prime} a$ and $v^{\prime} b$ such that $\tau^{\prime}=\pi\left(v^{\prime} a, m_{1}^{\prime}\right)$ and either $\pi\left(v^{\prime} a, m_{1}^{\prime}\right)=\pi\left(v^{\prime} b, m_{2}^{\prime}\right)$, or $\pi\left(v^{\prime} a, m_{1}^{\prime}\right) \sim \pi\left(v^{\prime} b, m_{2}^{\prime}\right)$. Hence $\pi\left(v a, m_{1}\right)=\pi\left(v b, m_{2}\right)$ due to Lemma 13 or Case 3 of Lemma 15. So $\Lambda_{v a}\left(\tau^{\prime}\right)=\tau \in K_{v}$ or $\Lambda_{v a}\left(\tau^{\prime}\right)=\tau \in R_{v}$. But we have already proved that $R_{v}=\emptyset$, so $\Lambda_{v a}\left(\tau^{\prime}\right) \in K_{v}$. Let $\tau_{1}^{\prime}$ and $\tau_{2}^{\prime}$ be two different elements of $K_{v^{\prime}} \cup T_{v^{\prime}} \cup R_{v^{\prime}}$. Then by the definition of $K_{v}, T_{v}$ and $R_{v}$ we have $\tau_{1}^{\prime} \nsim \tau_{2}^{\prime}$. Hence Case 3 of Lemma 7 implies that $\Lambda_{v a}\left(\tau_{1}^{\prime}\right) \neq \Lambda_{v a}\left(\tau_{2}^{\prime}\right)$. Hence $\Lambda_{v a}$ is an injective map from $K_{v^{\prime}} \cup T_{v^{\prime}} \cup R_{v^{\prime}}$ to $K_{v}$ and

$$
k_{v^{\prime}}+t_{v^{\prime}}+r_{v^{\prime}}=\left|K_{v^{\prime}} \cup T_{v^{\prime}} \cup R_{v^{\prime}}\right| \leq\left|K_{v}\right|=k_{v} .
$$

Combining the inequalities proven above, we obtain that $k_{v}=k_{v^{\prime}}+t_{v^{\prime}}+r_{v^{\prime}}$. Since by definition $g(v)=k_{v}+r_{v}$, and as $r_{v}=0$ we have $g(v)=k_{v^{\prime}}+t_{v^{\prime}}+r_{v^{\prime}}$.

Lemma 17 Let $v$ be a special word with the ancestor $v^{\prime}$ with $|v| \geq L_{\omega}$, and $|v|=l\left|v^{\prime}\right|$. Then $g(v)=$ $g\left(v^{\prime}\right)$.

Proof: Since $|v|=l\left|v^{\prime}\right|$, we obtain that $v$ and $v^{\prime}$ have the same first symbol $a$.

Let us prove $g\left(v^{\prime}\right) \leq g(v)$. For each permutation $\tau^{\prime}$ of $G_{v^{\prime}}$, consider arbitrary occurrences $\left(v^{\prime} a, m_{1}^{\prime}\right)$ and $\left(v^{\prime} b, m_{2}^{\prime}\right)$ of words $v^{\prime} a$ and $v^{\prime} b$ such that $\tau^{\prime}=\pi\left(v^{\prime} a, m_{1}^{\prime}\right)=\pi\left(v^{\prime} b, m_{2}^{\prime}\right)$. Let $\Lambda_{v a}\left(\tau^{\prime}\right)=\tau$. By Lemma 13 we have $\pi\left(v a, m_{1}\right)=\pi\left(v b, m_{2}\right)$. So $\tau \in G_{v}$. Let $\tau_{3}^{\prime}$ and $\tau_{4}^{\prime}$ be two different elements of $G_{v^{\prime}}$. Since $v b$ does not generate conjugate permutations, we have $\tau_{3}^{\prime} \nsim \tau_{4}^{\prime}$. Hence Case 3 of Lemma 7 implies that $\Lambda_{v a}\left(\tau_{3}^{\prime}\right) \neq \Lambda_{v a}\left(\tau_{4}^{\prime}\right)$. Hence $\Lambda_{v a}$ is an injective map from $G_{v^{\prime}}$ to $G_{v}$ and

$$
g\left(v^{\prime}\right)=\left|G_{v^{\prime}}\right| \leq\left|G_{v}\right|=g(v) .
$$

Let us prove $g(v) \leq g\left(v^{\prime}\right)$. For each permutation $\tau$ of $G_{v}$, consider arbitrary occurrences $\left(v a, m_{1}\right)$ and $\left(v b, m_{2}\right)$ of words $v a$ and $v b$ such that $\tau=\pi\left(v a, m_{1}\right)=\pi\left(v b, m_{2}\right)$. Let $\Psi_{v a}(\tau)=\tau^{\prime}$. Let us prove that $\tau^{\prime} \in G_{v^{\prime}}$. If $\pi\left(v^{\prime} a, m_{1}^{\prime}\right) \sim \pi\left(v^{\prime} b, m_{2}^{\prime}\right)$, then $\pi\left(v a, m_{1}\right) \sim \pi\left(v b, m_{2}\right)$ due to Case 1 of Lemma 15 . So $\pi\left(v^{\prime} a, m_{1}^{\prime}\right)=\pi\left(v^{\prime} b, m_{2}^{\prime}\right)$ due to Lemma 14 and $\Psi_{v a}(\tau)=\tau^{\prime} \in G_{v^{\prime}}$. Let $\tau_{3}$ and $\tau_{4}$ be two different elements of $G_{v}$. Hence Case 1 of Lemma 7 implies that $\Psi_{v a}\left(\tau_{3}\right) \neq \Psi_{v a}\left(\tau_{4}\right)$. Hence $\Psi_{v a}$ is an injective map from $G_{v}$ to $G_{v^{\prime}}$ and

$$
g(v)=\left|G_{v}\right| \leq\left|G_{v^{\prime}}\right|=g\left(v^{\prime}\right) .
$$

Lemma 18 Let $v$ be a special word with the ancestor $v^{\prime}$ such that $|v| \geq L_{\omega}$, and $|v|=l\left|v^{\prime}\right|$. Then $k_{v}+t_{v}+r_{v}=k_{v^{\prime}}+t_{v^{\prime}}+r_{v^{\prime}}$.

Proof: Since $|v|=l\left|v^{\prime}\right|$, we obtain that $v$ and $v^{\prime}$ have the same first symbol $a$. Let $b=\{0,1\} \backslash a$.

Let us prove that $t_{v^{\prime}} \leq t_{v}$. For each permutation $\tau^{\prime}$ of $T_{v^{\prime}}$, consider arbitrary occurrences $\left(v^{\prime} a, m_{1}^{\prime}\right)$ and $\left(v^{\prime} b, m_{2}^{\prime}\right)$ of words $v^{\prime} a$ and $v^{\prime} b$ such that $\tau^{\prime}=\pi\left(v^{\prime} a, m_{1}^{\prime}\right) \sim \pi\left(v^{\prime} b, m_{2}^{\prime}\right)$. Let $\Lambda_{v a}\left(\tau^{\prime}\right)=\tau$. Let us prove that $\tau \in T_{v}$. Indeed, Case 1 of Lemma 15 implies that $\pi\left(v a, m_{1}\right) \sim \pi\left(v b, m_{2}\right)$. Hence we have that either $\tau \in T_{v}$ or $\tau \sim \tau_{1}$ and $\tau_{1} \in R_{v}$. Consider the second case. Then there exists an occurrence $\left(v a, m_{3}\right)$ such that $\pi\left(v a, m_{1}\right) \sim \pi\left(v a, m_{3}\right)$. Hence Case 2 of Lemma 7 implies that $\pi\left(v^{\prime} a, m_{1}^{\prime}\right) \sim \pi\left(v^{\prime} a, m_{3}^{\prime}\right)$. It contradicts with that $\tau^{\prime}=\pi\left(v^{\prime} a, m_{1}^{\prime}\right) \in T_{v^{\prime}}$. Let $\tau_{3}^{\prime}$ and $\tau_{4}^{\prime}$ be two different elements of $T_{v^{\prime}}$. Then by 
definition of $T_{v^{\prime}}$ we have $\tau_{3}^{\prime} \nsim \tau_{4}^{\prime}$. Hence Case 3 of Lemma 7 implies that $\Lambda_{v a}\left(\tau_{3}^{\prime}\right) \neq \Lambda_{v a}\left(\tau_{4}^{\prime}\right)$. Hence $\Lambda_{v a}$ is an injective map from $T_{v^{\prime}}$ to $T_{v}$ and

$$
t_{v^{\prime}}=\left|T_{v^{\prime}}\right| \leq\left|T_{v}\right|=t_{v} .
$$

Let us prove that $t_{v} \leq t_{v^{\prime}}$. For each permutation $\tau$ of $T_{v}$, consider arbitrary occurrences $\left(v a, m_{1}\right)$ and $\left(v b, m_{2}\right)$ of words $v a$ and $v b$ such that $\tau=\pi\left(v a, m_{1}\right) \sim \pi\left(v b, m_{2}\right)$. Let $\Psi_{v a}(\tau)=\tau^{\prime}$. Let us prove that $\tau^{\prime} \in T_{v^{\prime}}$. Indeed, due to Case 2 of Lemma 15 we have $\pi\left(v^{\prime} a, m_{1}^{\prime}\right) \sim \pi\left(v^{\prime} b, m_{2}^{\prime}\right)$. Hence we have either $\Psi_{v a}(\tau)=\tau^{\prime} \in T_{v^{\prime}}$, or $\tau^{\prime} \sim \tau^{\prime \prime}$ and $\tau^{\prime \prime} \in R_{v^{\prime}}$. Consider the second case. Then there exists an occurrence $\left(v^{\prime} a, m_{3}^{\prime}\right)$ such that $\pi\left(v^{\prime} a, m_{1}^{\prime}\right) \sim \pi\left(v^{\prime} a, m_{3}^{\prime}\right)$. Hence Case 4 of Lemma 7 implies that $\pi\left(v a, m_{1}\right) \sim \pi\left(v b, m_{3}\right)$. It contradicts with $\tau \in T_{v}$. Let $\tau_{3}$ and $\tau_{4}$ be two different elements of $T_{v}$. Hence Case 1 of Lemma 7 implies that $\Psi_{v a}\left(\tau_{3}\right) \neq \Psi_{v a}\left(\tau_{4}\right)$. Hence $\Psi_{v a}$ is an injective map from $T_{v}$ to $T_{v^{\prime}}$ and

$$
t_{v}=\left|T_{v}\right| \leq\left|T_{v^{\prime}}\right|=t_{v^{\prime}} .
$$

So $t_{v}=t_{v^{\prime}}$. By Lemma 17 we have $g(v)=g\left(v^{\prime}\right)$. Since $g(v)=k_{v}+r_{v}$ and $g\left(v^{\prime}\right)=k_{v^{\prime}}+r_{v^{\prime}}$, we have

$$
k_{v}+t_{v}+r_{v}=k_{v^{\prime}}+t_{v^{\prime}}+r_{v^{\prime}} .
$$

Lemma 19 Let $n=x l+r \geq L_{\omega}$, where $1 \leq r \leq l$. Then the following statements are true:

1. $\alpha(n)=\beta(x+1)$ for $r<l$ and $\alpha((x+1) l)=\alpha(x+1)$.

2. $\beta(n)=\alpha(n)$ for $r<l$ and $\beta((x+1) l)=\beta(x+1)$.

Proof: Let $u \in B(n)$. Since $u$ is a special word with $|u| \geq L_{\omega}, u$ has unique interpretation $\langle v, i, 0\rangle$, where $0 \leq i<l$ and $v$ is the ancestor of $u$. By the definition of interpretation we have $|u|=|v| l-i$. Hence $i \equiv l-r(\bmod l)$. So $|v|=x+1$ and $i=l-r$.

Let $B_{1}=\left\{\varphi_{l-r ; 0}(v) \mid v \in B(x+1)\right\}$. Let us prove the following assertion:

Proposition $5 B(n)=B_{1}$.

Proof: Let us prove that $B_{1} \subseteq B(n)$. Let $v \in B(x+1)$. Then $v 0$ and $v 1$ are subwords of $\omega$. Since $\omega$ is a fixed point of morphism $\varphi, \varphi(v 0)$ and $\varphi(v 1)$ are a subwords of $\omega$. Since

$$
\varphi(v 0)=\varphi(v) \varphi(0)=\varphi(v) 0 \ldots
$$

and

$$
\varphi(v 1)=\varphi(v) \varphi(1)=\varphi(v) 1 \ldots,
$$

words $\varphi(v) 0$ and $\varphi(v) 1$ are subwords of $\omega$. By the definition of $\varphi_{i ; j}(v)$ we have that $\varphi_{l-r ; 0}(v)$ is a suffix of $\varphi(v)$. So $\varphi_{l-r ; 0}(v) 0$ and $\varphi_{l-r ; 0}(v) 1$ are subwords of $\omega$. Let $u=\varphi_{l-r ; 0}(v)$. Then

$$
|u|=|v| l-l+r=x l+r .
$$


Since $\varphi_{l-r ; 0}(v) 0$ and $\varphi_{l-r ; 0}(v) 1$ are subwords of $\omega$ and $|u|=x l+r=n$, we obtain that $u \in B(n)$. Thus $B_{1} \subseteq B(n)$.

Let us prove that $B(n) \subseteq B_{1}$. Let $u \in B(n)$. As we proved above, $u$ has unique interpretation $\langle v, l-r, 0\rangle$, where $v$ is the ancestor of $u$ and $|v|=x+1$. Then $u 0$ and $u 1$ have interpretations $\langle v 0, l-$ $r, l-1\rangle$ and $\langle v 1, l-r, l-1\rangle$. So $v 0$ and $v 1$ are ancestors of $u 0$ and $u 1$. Hence $v \in B(x+1)$. Since $u$ has interpretation $\langle v, l-r, 0\rangle, u=\varphi_{l-r ; 0}(v)$ due to the definition of $\varphi_{i ; j}(v)$. So $u \in B_{1}$ due to the definition of $B_{1}$. Thus $B(n) \subseteq B_{1}$ and $B(n)=B_{1}$.

\section{Proof of Lemma 19:}

1. Let $r<l$. We have $B(n)=B_{1}$ due to Proposition 5 . Then

$$
\alpha(n)=\sum_{u \in B(n)} g(u)=\sum_{u \in B_{1}} g(u)
$$

Let $u=\varphi_{l-r ; 0}(v) \in B_{1}$. Then $v$ is the ancestor of $u,|u|=n=x l+r$ and $|v|=x+1$. Then Lemma 16 implies that $g(u)=k_{v}+t_{v}+r_{v}$. So

$$
\sum_{u \in B_{1}} g(v)=\sum_{v \in B(x+1)} g\left(\varphi_{l-r ; 0}(v)\right)=\sum_{v \in B(x+1)}\left(k_{v}+t_{v}+r_{v}\right)=\beta(x+1) .
$$

Thus $\alpha(n)=\beta(x+1)$.

Let $r=l$. We have $B(n)=B_{1}$ due to Proposition 5 Then

$$
\alpha(n)=\sum_{u \in B(n)} g(u)=\sum_{u \in B_{1}} g(u)
$$

Let $u=\varphi_{0 ; 0}(v) \in B_{1}$. Then $v$ is the ancestor of $u,|u|=n=(x+1) l$ and $|v|=x+1$. Then Lemma 17 implies that $g(u)=g(v)$. So

$$
\sum_{u \in B_{1}} g(u)=\sum_{v \in B(x+1)} g\left(\varphi_{0 ; 0}(v)\right)=\sum_{v \in B(x+1)} g(v)=\alpha(x+1) .
$$

Thus $\alpha((x+1) l)=\alpha(x+1)$.

2. Let $r<l$. Consider an arbitrary special word $v$ of length $n$. Assume that $t_{v}>0$. Then due to Case 2 of Lemma 15 , the existence of two conjugate permutations $\pi\left(v 0, m_{1}\right)$ and $\pi\left(v 1, m_{2}\right)$ would imply that $|v|=\left|v^{\prime}\right| l$. It contradicts with $r<l$. So

$$
\sum_{z \in B(n)}\left(k_{z}+t_{z}+r_{z}\right)=\sum_{z \in B(n)}\left(k_{z}+r_{z}\right)=\sum_{z \in B(n)} g(z) .
$$

Thus $\beta(n)=\alpha(n)$ for $r<l$.

Let $r=l$. We have $B(n)=B_{1}$ due to Proposition 5 Then

$$
\beta(n)=\sum_{u \in B(n)}\left(k_{u}+t_{u}+r_{u}\right)=\sum_{u \in B_{1}}\left(k_{u}+t_{u}+r_{u}\right) .
$$


Let $u=\varphi_{0 ; 0}(v) \in B_{1}$. Then $v$ is the ancestor of $u,|u|=n=(x+1) l$ and $|v|=x+1$. Then Lemma 18 implies that $k_{u}+t_{u}+r_{u}=k_{v}+t_{v}+r_{v}$. So

$$
\sum_{u \in B_{1}}\left(k_{u}+t_{u}+r_{u}\right)=\sum_{v \in B(x+1)}\left(k_{u}+t_{u}+r_{u}\right)=\sum_{v \in B(x+1)}\left(k_{v}+t_{v}+r_{v}\right)=\beta(x+1) .
$$

Thus $\beta((x+1) l)=\beta(x+1)$.

Lemma 20 Let $n \geq L_{\omega}$. Then $\beta(n)=\alpha(n)$ for $r(n)<l^{s(n)}$ and $\beta(n)=\beta(k(n)+1)$ for $r(n)=l^{s(n)}$.

Proof: Let us prove by the induction on $s(n)$ that $\beta(n)=\alpha(n)$ for $r(n)<l^{s(n)}$. For $s(n)=1$ it is true due to Case 2 of Lemma 19. Let us prove the induction step. Let $n=x l+r_{0}$ for $r_{0} \in[1, l]$. If $r_{0}<l$, then $\beta(n)=\alpha(n)$ due to Case 2 of Lemma 19 . Consider the case when $r_{0}=l$. Then $n=(x+1) l$ and we have $\beta((x+1) l)=\beta(x+1)$ due to Case 2 of Lemma 19 Since

$$
k(n) l^{s(n)}+1 \leq n \leq(k(n)+1) l^{s(n)}-1,
$$

we have $x+1 \in\left[k(n) l^{s(n)-1}+1,(k(n)+1) l^{s(n)-1}-1\right]$. Then by the induction assumption we have that $\beta(x+1)=\alpha(x+1)$. Hence $\beta((x+1) l)=\alpha(x+1)$. Since $\alpha((x+1) l)=\alpha(x+1)$ due to Case 1 of Lemma 19, we obtain that $\beta((x+1) l)=\alpha((x+1) l)$.

Let us prove by the induction on $s(n)$ that $\beta\left((k(n)+1) l^{s(n)}\right)=\beta(k(n)+1)$. For $s(n)=0$ it is true. Let us prove the induction step. We have $\beta(x l)=\beta(x)$ due to Case 2 of Lemma 19 By the induction assumption we have that $\beta\left((k(n)+1) l^{s(n)-1}\right)=\beta(k(n)+1)$. Hence $\beta\left((k(n)+1) l^{s}(n)\right)=\beta(k(n)+1)$.

\section{Proof of Theorem 2 ;}

Let us prove that $\alpha\left((k(n)+1) l^{s(n)}\right)=\alpha(k(n)+1)$ by the induction on $s(n)$. For $s(n)=1$ it is true due to Case 1 of Lemma 19 . Let us prove the induction step. We have

$$
\alpha\left((k(n)+1) l^{s(n)}\right)=\alpha\left((k(n)+1) l^{s(n)-1}\right)
$$

due to Lemma 19 By the induction assumption we have $\alpha\left((k(n)+1) l^{s(n)-1}\right)=\alpha(k(n)+1)$. So

$$
\alpha(n)=\alpha(k(n)+1)
$$

for $r(n)=l^{s(n)}$.

Let us prove that $\alpha(n)=\beta(k(n)+1)$ for $r(n)<l^{s(n)}$. For $s(n)=1$ it is true due to Case 1 of Lemma 19. Let us prove the induction step. Let $n=x l+r_{0}$ for $r_{0} \in[1, l]$. Consider the case when $r_{0}<l$. Since

$$
k(n) l^{s(n)}+1 \leq n \leq(k(n)+1) l^{s(n)}-1,
$$

we have

$$
k(n) l^{s(n)-1}+\frac{1-l}{l} \leq x \leq(k(n)+1) l^{s(n)-1}-\frac{2}{l} .
$$

Hence

$$
k(n) l^{s(n)-1} \leq x \leq(k(n)+1) l^{s(n)-1}-1 .
$$


Hence $x+1 \in\left[k(n) l^{s(n)-1}+1,(k(n)+1) l^{s(n)-1}\right]$. If $x+1 \in\left[k(n) l^{s(n)-1}+1,(k(n)+1) l^{s(n)-1}-1\right]$, then $\beta(x+1)=\alpha(x+1)$ due to Lemma 20 We have $\alpha(n)=\beta(x+1)$ due to Case 1 of Lemma 19 . Hence $\alpha(n)=\alpha(x+1)$. By the induction assumption we have $\alpha(x+1)=\beta(k(n)+1)$. So $\alpha(n)=\beta(k(n)+1)$. It remains to consider the case when $x+1=(k(n)+1) l^{s(n)-1}$. Then we have $\beta(x+1)=\beta(k(n)+1)$ due to Lemma 20 Hence $\alpha(n)=\beta(x+1)=\beta(k(n)+1)$.

Consider the case when $r_{0}=l$. Then $n=(x+1) l$ and we have $\alpha(n)=\alpha(x+1)$ due to Lemma 19 Since

$$
k(n) l^{s(n)}+1 \leq n \leq(k(n)+1) l^{s(n)}-1,
$$

we have

$$
k(n) l^{s(n)-1}+1 \leq x+1 \leq(k(n)+1) l^{s(n)-1}-1 .
$$

Then $\alpha(x+1)=\beta(k(n)+1)$ due to the induction assumption. So $\alpha(n)=\beta(k(n)+1)$.

\section{Computing the permutation complexity}

In this section we state the main theorem of this article. Let $n \geq L_{\omega}$. Then for $n$ there exists a unique pair of numbers $k(n)$ and $s(n)$ such that

$$
s(n)>0, k(n) \in\left\{l, \ldots, l^{2}-1\right\}
$$

and

$$
k(n) l^{s(n)}<n \leq(k(n)+1) l^{s(n)} .
$$

The number $n-k(n) l^{s(n)}$ is denoted by $r(n)$. We note that $n=k(n) l^{s(n)}+r(n)$ and $r(n) \in\left[1 ; l^{s(n)}\right]$.

Recall that $\mu(n)=\sum_{|u|=n}\left(m_{u}+n_{u}\right), \chi(n)=\sum_{|u|=n} f(u), \alpha(n)=\sum_{z \in B(n)} g(z)$ and $\beta(n)=$ $\sum_{z \in B(n)}\left(k_{z}+t_{z}+r_{z}\right)$.

Theorem 3 Let $\omega$ be a fixed point of the morphism $\varphi$, where $\varphi \in Q_{l}$ and $n \geq L_{\omega}$. Then the permutation complexity of $\omega$ is calculated as follows:

$$
\lambda(n)=(r(n)-1) \mu(k(n)+2)+\left(l^{s(n)}-r(n)+1\right) \chi(k(n)+1)-\beta(k(n)+1)
$$

for $r(n)>1$ and

$$
\lambda(n)=l^{s(n)} \chi(k(n)+1)-\alpha(k(n))
$$

for $r(n)=1$.

Proof: We note that $\sum_{|u|=n} f(u)$ is the number of permutations, each of them is generated by at least one occurrence of some subword of word $\omega$ of length $n$. It is clear that some permutations can be calculated several times. But Lemma 12 implies that two distinct subwords $u_{1}$ and $u_{2}$ of word $\omega$ can generate the same permutations if and only if $u_{1}=v 0$ and $u_{2}=v 1$ up to a relabeling of $u_{1}$ and $u_{2}$. Hence only $g(v)$ permutations are calculated two times in the sum $\sum_{|u|=n} f(u)$. Thus

$$
\lambda(n)=\sum_{|u|=n} f(u)-\sum_{b \in B(n-1)} g(b) .
$$

Combining Theorem 1 and 2 we prove the main theorem. 
Now for the calculation of $\lambda(n)$ it remains to compute $\mu(k(n)+2), \chi(k(n)+1), \beta(k(n)+1)$ and $\alpha(k(n))$ for $k(n) \in\left\{l, \ldots, l^{2}-1\right\}$. Thus it is sufficient to find the set $H_{u}$ (the set of permutations generated by $u$ ) for any subword $u$ of $\omega$ with $|u| \in\left\{l+1, \ldots, l^{2}+1\right\}$.

Proposition 6 Let $\varphi \in Q_{l}$ and $\omega=\varphi(\omega)$. Then $\omega$ is $l^{2}+1$-free.

Proof: Let us prove that $v^{l^{2}+1}$ is not subword of $\omega$ by the induction on $|v|$. If $|v|=1$, then $v$ is some letter, i.e, $a^{l^{2}+1}$ is the subword of $\omega$ for some $a \in\{0,1\}$. Since $l^{2}+1>2 l-1$, we have that $\varphi(b)$ is the subword $a^{l^{2}+1}$ for some $b \in\{0,1\}$. Hence $\varphi(b)=a^{l}$ and we obtain contradiction (otherwise Properties in the definition of $Q_{l}$ do not hold).

Let us prove the induction step. Assume that $v^{l^{2}+1}$ is a subword of $\omega$. Let $v_{1}=v_{2}=\ldots=v_{l^{2}+1}=v$. Since $|v| \geq 2$ and $2 l^{2} \geq l^{2}+1$, we obtain that words $v_{1} v_{2} \ldots v_{l^{2}}$ and $v_{2} v_{3} \ldots v_{l^{2}+1}$ have the same partitions into blocks. From this partitions we have that $v_{1}=s_{1} \varphi(x) s_{2}$, where $x$ is a some word, $s_{1}$ is the suffix of $\varphi(a)$ and $s_{2}$ is the prefix of $\varphi(b)$ for $a, b \in\{0,1\}$. Since $v_{1} v_{2} \ldots v_{l^{2}}$ and $v_{2} \ldots v_{l^{2}+1}$ have the same partitions into blocks, we have that $v_{2}=s_{1} \varphi(x) s_{2}$. Moreover $s_{2} s_{1}=\varphi(c)$ for some $c \in\{0,1\}$. Continuing the procedure, we obtain that

$$
v_{1}=v_{2}=\ldots=v_{l^{2}+1}=s_{1} \varphi(x) s_{2} .
$$

So

$$
v^{l^{2}+1}=v_{1} \ldots v_{l^{2}+1}=s_{1} \varphi(x) s_{2} s_{1} \varphi(x) s_{2} \ldots s_{1} \varphi(x) s_{2}
$$

is the subword of $\omega$. Then $(c x)^{l^{2}+1}$ also is the subword of $\omega$. Since $|c x|<|v|$, we obtain a contradiction.

We find $H_{u}$ by Algorithm 1:

\section{Algorithm 1.}

1. We consider all occurrences $(u, m)$ of word $u$ in word $\varphi^{5}(0)$.

2. Let $1 \leq i<j \leq|u|$. Since $\omega$ is $l^{2}+1$-free, we have that words $\omega_{m+i} \ldots \omega_{m+i+(j-i) l^{2}-1}$ and $\omega_{m+j} \ldots \omega_{m+j+(j-i) l^{2}-1}$ are distinct. Let

$$
u_{i}=\omega_{m+i} \ldots \omega_{m+i+(|u|-1) l^{2}-1}
$$

and

$$
u_{j}=\omega_{m+j} \ldots \omega_{m+j+(|u|-1) l^{2}-1} .
$$

Then $u_{i} \neq u_{j}$. Since

$$
R_{\omega}(m+i)=0, u_{i} \ldots
$$

and

$$
R_{\omega}(m+j)=0, u_{j} \ldots
$$

we obtain that $R_{\omega}(m+i)>R_{\omega}(m+j)$ if and only if $u_{i}>u_{j}$ due to Proposition2 2 So if we know word

$$
\omega_{m+1} \ldots \omega_{m+(|u|-1)\left(l^{2}+1\right)},
$$

then we can find relation $\gamma\left(R_{\omega}(m+i), R_{\omega}(m+j)\right)$. Thus we find permutation $\pi(u, m)$. 
Proposition 7 Let $\left(u, m_{1}\right)$ and $\left(u, m_{2}\right)$ be some occurrences of $u$ such that $m_{1} \equiv m_{2}(\bmod l),\left(u^{\prime}, m_{1}^{\prime}\right)$ and $\left(u^{\prime}, m_{2}^{\prime}\right)$ be ancestors of $\left(u, m_{1}\right)$ and $\left(u, m_{2}\right)$. Then if $\pi\left(u^{\prime}, m_{1}^{\prime}\right)=\pi\left(u^{\prime}, m_{2}^{\prime}\right)$, then $\pi\left(u, m_{1}\right)=$ $\pi\left(u, m_{2}\right)$.

Proof: Consider relations $\gamma\left(R_{\omega}\left(m_{1}+t\right), R_{\omega}\left(m_{1}+s\right)\right)$ and $\gamma\left(R_{\omega}\left(m_{2}+t\right), R_{\omega}\left(m_{2}+s\right)\right)$ for $1 \leq t<$ $s \leq|u|$. If $\omega_{m_{1}+t} \neq \omega_{m_{1}+s}$, then $\omega_{m_{2}+t} \neq \omega_{m_{2}+s}$. Hence

$$
\gamma\left(R_{\omega}\left(m_{1}+t\right), R_{\omega}\left(m_{1}+s\right)\right)=\gamma\left(\omega_{m_{1}+t}, \omega_{m_{1}+s}\right)
$$

and

$$
\gamma\left(R_{\omega}\left(m_{2}+t\right), R_{\omega}\left(m_{2}+s\right)\right)=\gamma\left(\omega_{m_{2}+t}, \omega_{m_{2}+s}\right) .
$$

So

$$
\gamma\left(R_{\omega}\left(m_{1}+t\right), R_{\omega}\left(m_{1}+s\right)\right)=\gamma\left(R_{\omega}\left(m_{2}+t\right), R_{\omega}\left(m_{2}+s\right)\right) .
$$

Consider the case when $\omega_{m_{1}+t}=\omega_{m_{1}+s}$ and $\omega_{m_{2}+t}=\omega_{m_{2}+s}$. Let $u_{1}=\omega_{m_{1}+1} \ldots \omega_{m_{1}+n}$ and $u_{2}=\omega_{m_{2}+1} \ldots \omega_{m_{2}+n}$. Since $\left(u^{\prime}, m_{1}^{\prime}\right)$ and $\left(u^{\prime}, m_{2}^{\prime}\right)$ are ancestors of $\left(u, m_{1}\right)$ and $\left(u, m_{2}\right)$ and $m_{1} \equiv m_{2}$ $(\bmod l)$, words $u_{1}$ and $u_{2}$ have the same partitions in the correct partition of $\omega$. Hence $\omega_{m_{1}+t}, \omega_{m_{1}+s}$, $\omega_{m_{2}+t}$ and $\omega_{m_{2}+s}$ lie in blocks $\varphi\left(\omega_{m_{1}^{\prime}+t^{\prime}}\right), \varphi\left(\omega_{m_{1}^{\prime}+s^{\prime}}\right), \varphi\left(\omega_{m_{2}^{\prime}+t^{\prime}}\right)$ and $\varphi\left(\omega_{m_{2}^{\prime}+s^{\prime}}\right)$ in the correct partition of $\omega$ for some $1 \leq t^{\prime}<s^{\prime} \leq\left|u^{\prime}\right|$. Moreover $\omega_{m_{1}^{\prime}+t^{\prime}}=\omega_{m_{2}^{\prime}+t^{\prime}}$ and $\omega_{m_{1}^{\prime}+s^{\prime}}=\omega_{m_{2}^{\prime}+s^{\prime}}$. Let

$$
\omega_{m_{1}^{\prime}+t^{\prime}}=\omega_{m_{2}^{\prime}+t^{\prime}}=a
$$

and

$$
\omega_{m_{1}^{\prime}+s^{\prime}}=\omega_{m_{2}^{\prime}+s^{\prime}}=b .
$$

Since $m_{1} \equiv m_{2}(\bmod l)$, we have $m_{1}+t \equiv m_{2}+t(\bmod l)$ and $m_{1}+s \equiv m_{2}+s(\bmod l)$. Applying Lemma 2 to $\left(u, m_{1}\right)$ and $\left(u, m_{2}\right)$, we obtain that

$$
\gamma\left(R_{\omega}\left(m_{1}+t\right), R_{\omega}\left(m_{1}+s\right)\right)=\gamma\left(R_{\omega}\left(m_{2}+t\right), R_{\omega}\left(m_{2}+s\right)\right)
$$

in the case when $a \neq b$ or $s \not \equiv t(\bmod l)$. In the case when $a=b$ and $s \equiv t(\bmod l)$ we have

$$
\gamma\left(R_{\omega}\left(m_{1}+t\right), R_{\omega}\left(m_{1}+s\right)\right)=\gamma\left(R_{\omega}\left(m_{1}^{\prime}+t^{\prime}\right), R_{\omega}\left(m_{1}^{\prime}+s^{\prime}\right)\right)
$$

and

$$
\gamma\left(R_{\omega}\left(m_{2}+t\right), R_{\omega}\left(m_{2}+s\right)\right)=\gamma\left(R_{\omega}\left(m_{2}^{\prime}+t^{\prime}\right), R_{\omega}\left(m_{2}^{\prime}+s^{\prime}\right)\right) .
$$

Since $\pi\left(u^{\prime}, m_{1}^{\prime}\right)=\pi\left(u^{\prime}, m_{2}^{\prime}\right)$, we have

$$
\gamma\left(R_{\omega}\left(m_{1}+t\right), R_{\omega}\left(m_{1}+s\right)\right)=\gamma\left(R_{\omega}\left(m_{2}+t\right), R_{\omega}\left(m_{2}+s\right)\right)
$$

and $\pi\left(u, m_{1}\right)=\pi\left(u, m_{2}\right)$.

Lemma 21 1. Let $\left(u, m_{1}\right)$ and $\left(u, m_{2}\right)$ be two occurrences of $u$ of length at most 2 . Then $\pi\left(u, m_{1}\right)=$ $\pi\left(u, m_{2}\right)$.

2. $\varphi^{3}(0)$ contains all subwords of word $\omega$ of length 2 . 
3. Let $\pi \in H_{u}$ and $|u| \leq l+1$. Then there exists an occurrence $(u, m)$ of $u$ such that $\pi(u, m)=\pi$ and $(u, m)$ is an occurrence of $u$ in $\varphi^{4}(0)$.

4. Let $\pi \in H_{u}$ and $|u| \in\left\{l+1, \ldots, l^{2}+1\right\}$. Then there exists an occurrence $(u, m)$ of $u$ such that $\pi(u, m)=\pi$ and $(u, m)$ is an occurrence of $u$ in $\varphi^{5}(0)$.

\section{Proof:}

1. The case when $|u|=1$ is an obvious because $\pi(u, m)=1$ for any occurrence $(u, m)$. Consider the case when $|u|=2$. Let $u=a b$. If $a \neq b$, then $\gamma(R(m+1), R(m+2))=\gamma(a, b)$ for any occurrence $(u, m)$. Thus we have $\pi\left(u, m_{1}\right)=\pi\left(u, m_{2}\right)$ for $a \neq b$.

Consider the case when $a=b$. Let $a=0$ (the case when $a=1$ is similar). Let us prove that $\gamma(R(m+1), R(m+2))=<$ for any occurrence $(u, m)$. Let $t$ be the minimal number such that $\omega_{t}=1$ and $t>m+1$ (it exists because word $\omega$ is nonperiodic). Then

$$
\gamma\left(R_{\omega}(m+1), R_{\omega}(m+2)\right)=\gamma\left(0,0^{t-m-1} \ldots, 0,0^{t-m-2} 1 \ldots\right)=<.
$$

So $\pi(u, m)=12$ for any occurrence $(u, m)$. Thus $\pi\left(u, m_{1}\right)=\pi\left(u, m_{2}\right)$.

2. Since $\varphi \in Q_{l}$, we have that $\varphi$ is a marked morphism and $\varphi(0)$ starts with 0 . Since $\varphi$ is a marked morphism, $\varphi(1)$ starts with 1 . Let $\varphi(0)=0^{k} 1 \ldots$ for $k>1$ or $\varphi(0)=0^{l}$. If $\varphi(0)=0^{k} 1 \ldots$, then $0 u 1$ is a subword of $\varphi(0)$ and $0 u 0$ is a prefix of $\varphi(0)$ for empty word $u$. It contradicts with Property 1 in the definition of $Q_{l}$. If $\varphi(0)=0^{l}$, then $0 u$ is a suffix of $\varphi(0)$ and $0 u 0$ is a prefix of $\varphi(0)$ for empty word $u$. It contradicts with Property 1 in the definition of $Q_{l}$. Thus $\varphi(0)$ starts with 01 . Analogously one can obtain that $\varphi(1)$ starts with 10 . Since 01 is a subword of $\varphi(0), \varphi(01)$ is a subword of $\varphi^{2}(0)$. But we have

$$
\varphi(01)=\varphi(0) \varphi(1)=01 \ldots 10 \ldots
$$

So 01 and 10 are subwords of $\varphi^{2}(0)$. Let $\varphi(0)=0 x a$ and $\varphi(1)=1 y b$ for some words $x, y$ and letters $a$ and $b$. Since $\varphi$ is a marked morphism, $a \neq b$. Consider the case when $a=1$ and $b=0$. Then

$$
\varphi(01)=\varphi(0) \varphi(1)=\ldots 11 \ldots
$$

and

$$
\varphi(10)=\varphi(0) \varphi(1)=\ldots 00 \ldots
$$

So 00 and 11 are subwords of $\varphi^{3}(0)$.

It remains to consider the case when $a=0$ and $b=1$. Let word $a a$ be a subword of $\omega$. Let $i$ be the minimal number such that $\omega_{i} \omega_{i+1}=a a$. Let $u=\omega_{i} \omega_{i+1}$. If $u$ lies in block $\varphi(c)$ for some $c \in\{0,1\}$ in the correct partition of $\omega$ into blocks, then $u$ is a subword of $\varphi(c)$. Then $\varphi^{2}(c)=\varphi(c) \ldots$ and $u$ is a subword of $\varphi^{2}(c)$. Since $c$ is a subword of $\varphi(0), u$ is a subword of $\varphi^{3}(0)$. It remains the case when $\omega_{i}$ and $\omega_{i+1}$ lie in blocks $\varphi\left(\omega_{j}\right)$ and $\varphi\left(\omega_{j+1}\right)$ in the correct partition of $\omega$ into blocks. Then $\varphi\left(\omega_{j}\right)$ ends with $a$ and $\varphi\left(\omega_{j+1}\right)$ starts with $a$. Hence $\omega_{j}=\omega_{j+1}=a$. So $\omega_{j} \omega_{j+1}=a a$ and $j<i$. It contradicts with the minimality of $i$. Thus if $a a$ is a subword of $\omega$, then $a a$ is a subword of $\varphi^{3}(0)$.

3. Let $|u|=k$. Since $\pi \in H_{u}$, there exists some occurrence $\left(u, m_{1}\right)$ of $u$ such that $\pi\left(u, m_{1}\right)=\pi$. Let $\left(u_{m_{1}}^{\prime}, m_{1}^{\prime}\right)$ be the ancestor of $\left(u, m_{1}\right)$. Since $|u| \leq l+1$, we have that either $\omega_{m_{1}+1} \ldots \omega_{m_{1}+k}=s p$, where $s$ is a suffix of $\varphi\left(\omega_{i}\right), p$ is a prefix of $\varphi\left(\omega_{i+1}\right)$ for some $i$, or $\omega_{m_{1}+1} \ldots \omega_{m_{1}+k}$ is a subword of $\varphi\left(\omega_{i}\right)$ for some $i$. Consider the first case. Then $m_{1}=i l-|s|$. Let $\omega_{i}=a$ and $\omega_{i+1}=b$. Then 
$u_{m_{1}}^{\prime}=a b$ due to the definition of the ancestor of occurrence. Since $\varphi^{3}(0)$ contain all subwords of word $\omega$ of length 2 , there exists $m^{\prime}$ such that $\omega_{m^{\prime}+1} \omega_{m^{\prime}+2}=a b$ and $\omega_{m^{\prime}+1} \omega_{m^{\prime}+2}$ is a subword of $\varphi^{3}(0)$. Since $\omega=\varphi(\omega)$, we have $\omega_{\left(m^{\prime}+1\right) l-|s|} \ldots \omega_{\left(m^{\prime}+1\right) l-|s|+k-1}=s p$. Thus $\left(a b, m^{\prime}\right)$ is the ancestor of $(u, m)$ due to the definition of the ancestor of occurrence, where $m=\left(m^{\prime}+1\right) l-|s|$. Moreover $m \equiv m_{1}(\bmod l)$. We have $\pi\left(a b, m^{\prime}\right)=\pi\left(a b, m_{1}^{\prime}\right)$ due to Case 1 of this lemma. Since $m \equiv m_{1}(\bmod l)$, Proposition 7 implies that $\pi(u, m)=\pi\left(u, m_{1}\right)$. The case when $\omega_{m_{1}+1} \ldots \omega_{m_{1}+k}$ is a subword of $\varphi\left(\omega_{i}\right)$ for some $i$ is similar. Since $\left(u_{m_{1}}^{\prime}, m^{\prime}\right)$ is an occurrence of $u_{m_{1}}^{\prime}$ in $\varphi^{3}(0),(u, m)$ is an occurrence of $u$ in $\varphi^{4}(0)$.

4. Since $\pi \in H_{u}$, there exists some occurrence $\left(u, m_{1}\right)$ of $u$ such that $\pi\left(u, m_{1}\right)=\pi$. Let $\left(u_{m_{1}}^{\prime}, m_{1}^{\prime}\right)$ be the ancestor of $\left(u, m_{1}\right)$. Since $|u| \leq l^{2}+1$, we have that $\left|u_{m_{1}}^{\prime}\right| \leq l+1$. Then there exists an occurrence $\left(u_{m_{1}}^{\prime}, m^{\prime}\right)$ of $u_{m_{1}}^{\prime}$ in $\varphi^{4}(0)$ such that $\pi\left(u_{m_{1}}^{\prime}, m^{\prime}\right)=\pi\left(u_{m_{1}}^{\prime}, m_{1}^{\prime}\right)$ due to Case 3 of this Lemma. Let $m=$ $m^{\prime} l+r$, where $r$ is the residue of $m_{1}$ modulo $l$. Since $\omega=\varphi(\omega)$, we have $\omega_{m^{\prime} l+r} \ldots \omega_{m^{\prime} l+r+|u|-1}=$ $u$. Thus $\left(u_{m}^{\prime}, m^{\prime}\right)$ is the ancestor of $(u, m)$ due to the definition of the ancestor of occurrence. Then Proposition 7 implies that $\pi(u, m)=\pi\left(u, m_{1}\right)$. Since $\left(u_{m_{1}}^{\prime}, m^{\prime}\right)$ is an occurrence of $u_{m_{1}}^{\prime}$ in $\varphi^{4}(0)$, $(u, m)$ is an occurrence of $u$ in $\varphi^{5}(0)$.

The correctness of Algorithm 1. By Lemma21, we have that for any permutation $\pi$ from the set $H_{u}$ there exists an occurrence $(u, m)$ of word $u$ such that $\pi=\pi(u, m)$ and $\omega_{m+1} \omega_{m+2} \ldots \omega_{m+|u|}$ is a subword of $\varphi^{5}(0)$. Thus Algorithm 1 is correct.

\section{Permutation complexity of the Thue-Morse Word}

Widmer (2011) calculated the factor complexity of the permutation generated by the Thue-Morse word. In this section, we present an alternative proof for his formula. Recall that Thue-Morse word is a fixed point $\omega=\varphi(\omega)$ of morphism $\varphi(0)=01, \varphi(1)=10$. In what follows in this section $\omega$ is the Thue-Morse word.

As we note in previous section for the calculation of $\lambda(n)$ it is sufficient to find the set $H_{u}$ for any subword $u$ of $\omega$ with $|u| \in\left\{l+1, \ldots, l^{2}+1\right\}$. Since for Thue-Morse word $l=2$, it is sufficient to find the set $H_{u}$ for any subword $u$ of $\omega$ with $|u| \in\{3,4,5\}$. It can be found by Algorithm 1 . We consider in detail Algorithm 1 for words 010 and 01100 . We consider word

$$
\varphi^{5}(0)=011010 \underbrace{01100}_{(u, 6)} 101101001 \underbrace{01100}_{(u, 20)} 1101001 .
$$

An word 010 has two occurrences in $\varphi^{5}(0)$. It is $(010,3)$ and $(010,10)$. Now we find $\pi(010,3)$ and $\pi(010,10)$.

Permutation $\pi(010,3)$.

We have $\omega_{4} \ldots \omega_{13}=0100110010$. Hence $R_{\omega}(4)=0,0100110010 \ldots, R_{\omega}(5)=0,100110010 \ldots$ and $R_{\omega}(6)=0,00110010 \ldots$. So $R_{\omega}(6)<R_{\omega}(4)<R_{\omega}(5)$. Thus $\pi(010,3)=231$.

Permutation $\pi(010,10)$.

We have $\omega_{11} \ldots \omega_{20}=0101101001$. Hence $R_{\omega}(11)=0,0101101001 \ldots, R_{\omega}(12)=0,101101001 \ldots$ and $R_{\omega}(13)=0,01101001 \ldots$. So $R_{\omega}(12)<R_{\omega}(14)<R_{\omega}(13)$. So $\pi(010,10)=132$.

An word 01100 has two occurrences in $\varphi^{5}(0)$. It is $(01100,6)$ and $(01100,20)$. Now we find $\pi(01100,6)$ and $\pi(01100,20)$. 
Permutation $\pi(01100,6)$.

We have $\omega_{7} \ldots \omega_{26}=01100101101001011001$. Hence $R_{\omega}(7)=0,01100101101001011001 \ldots, R_{\omega}(8)=$ $0,1100101101001011001 \ldots, R_{\omega}(9)=0,100101101001011001 \ldots, R_{\omega}(10)=0,00101101001011001 \ldots$ and $R_{\omega}(11)=0,0101101001011001 \ldots$. So $\pi(01100,6)=35412$.

Permutation $\pi(01100,20)$.

We have $\omega_{21} \ldots \omega_{40}=01100110100110010110$. Hence $R_{\omega}(21)=0,01100110100110010110 \ldots, R_{\omega}(22)=$ $0,1100110100110010110 \ldots, R_{\omega}(23)=0,100110100110010110 \ldots, R_{\omega}(24)=0,00110100110010110 \ldots$ and $R_{\omega}(25)=0,0110100110010110 \ldots$. So $\pi(01100,20)=25413$.

Thus we obtain that $H_{01100}=\{25413,35412\}$.

For other subwords $u$ of $\omega$ with $|u| \in\{3,4,5\}$ we analogously find $H_{u}$ by the Algorithm 1 .

\section{Subpermutations of length 3 .}

$F_{\omega}(3)=\{001,010,011,100,101,110\}$. We have $H_{001}=\{123\}, H_{010}=\{132,231\}, H_{011}=\{132\}$, $H_{100}=\{312\}, H_{101}=\{213,312\}$ and $H_{110}=\{321\}$. Thus $\chi(3)=\sum_{|u|=3} f(u)=\sum_{|u|=3}\left|H_{u}\right|=8$. We have $B_{\omega}(2)=\{01,10\}$. Since $H_{010}=\{132,231\}$ and $H_{011}=\{132\}$, we have $g(01)=1$. Since $H_{100}=\{312\}$ and $H_{101}=\{213,312\}$, we have $g(10)=1$. So

$$
\alpha(2)=\sum_{v \in B(2)} g(v)=2 .
$$

\section{Subpermutations of length 4 .}

$F_{\omega}(4)=\{0101,0110,1001,1010,1011,1100,1101,0010,0011,0100\}$. We have $H_{0101}=\{1324\}$, $H_{0110}=\{2431\}, H_{1001}=\{3124\}, H_{1010}=\{4231\}, H_{1011}=\{3142\}, H_{1100}=\{4312\}, H_{1101}=$ $\{4312\}, H_{0010}=\{1243\}, H_{0011}=\{1243\}$ and $H_{0100}=\{2413\}$. Hence $m_{u}=1$ and $n_{u}=0$ for arbitrary subword $u$ of $\omega$ of length 4 . So

$$
\mu(4)=\sum_{|u|=4}\left(m_{u}+n_{u}\right)=10
$$

and

$$
\chi(4)=\sum_{|u|=4} f(u)=10 .
$$

We have $B_{\omega}(3)=\{001,010,101,110\}$. Since $H_{0010}=\{1243\}$ and $H_{0011}=\{1243\}$, we have $g(001)=$ $k_{001}=1$ and $t_{001}=r_{001}=0$. Since $H_{0100}=\{2413\}$ and $H_{0101}=\{1324\}$, we have $g(010)=k_{010}=$ $t_{010}=r_{010}=0$. Since $H_{1010}=\{4231\}$ and $H_{1011}=\{3142\}$, we have $g(101)=k_{101}=t_{101}=r_{101}=$ 0 . Since $H_{1100}=\{4312\}$ and $H_{1101}=\{4312\}$, we have $g(110)=k_{110}=1$ and $t_{110}=r_{110}=0$. So

$$
\alpha(3)=\sum_{v \in B(3)} g(v)=2
$$

and

$$
\beta(3)=\sum_{v \in B(3)}\left(k_{v}+t_{v}+r_{v}\right)=2 .
$$




\section{Subpermutations of length 5 .}

$F_{\omega}(5)=\{01011,10110,01100,11001,01101,11010,10010,00101,10011,00110,10100,01001\}$.

We have $H_{01011}=\{14253\}, H_{10110}=\{42531\}, H_{01100}=\{25413,35412\}, H_{11001}=\{43125,53124\}$,

$H_{01101}=\{25413\}, H_{11010}=\{54321\}, H_{10010}=\{41253\}, H_{00101}=\{12435\}, H_{10011}=\{31254,41253\}$,

$H_{00110}=\{13542,23541\}, H_{10100}=\{52413\}$ and $H_{01001}=\{24135\}$. Hence $m_{01100}=m_{10011}=0$, $n_{01100}=n_{10011}=1$ and $m_{u}=1, n_{u}=0$ for other subwords $u$ of $\omega$ of length 5 . So

$$
\mu(5)=\sum_{|u|=5}\left(m_{u}+n_{u}\right)=12 .
$$

We have $B_{\omega}(4)=\{0110,1001\}$. Since $H_{01100}=\{25413,35412\}$ and $H_{01101}=\{25413\}$, we have $k_{0110}=t_{0110}=0$ and $r_{0110}=1$. Since $H_{10010}=\{41253\}$ and $H_{10011}=\{31254,41253\}$, we have $k_{1001}=t_{1001}=0$ and $r_{1001}=1$. So

$$
\beta(4)=\sum_{v \in B(4)}\left(k_{v}+t_{v}+r_{v}\right)=2 .
$$

Thus if $n \geq 5$ and $n \in\left[2^{s+1}+1,3 \cdot 2^{s}\right]$, then $k(n)=2, s(n)=s$ and we have

$$
\lambda(n)=(r(n)-1) \mu(4)+\left(2^{s}-r(n)+1\right) \chi(3)-\beta(3)
$$

for $r>1$ and

$$
\lambda(n)=2^{s} \chi(3)-\alpha(2)
$$

for $r=1$ due to Theorem 3 . So in this case

$$
\lambda(n)=10(r(n)-1)+8\left(2^{s}-r(n)+1\right)-2=2\left(2^{s+2}+r(n)-2\right)
$$

for $r(n)>1$ and

$$
\lambda(n)=8 \cdot 2^{s}-2=2\left(2^{s+2}-1\right)
$$

for $r(n)=1$.

For $3 \cdot 2^{s}+1 \leq n \leq 2^{s+2}$ we have $k(n)=3, s(n)=s$ and Theorem 3 implies that

$$
\lambda(n)=(r(n)-1) \mu(5)+\left(2^{s}-r(n)+1\right) \chi(4)-\beta(4)
$$

for $r>1$ and

$$
\lambda(n)=2^{s} \chi(4)-\alpha(3)
$$

for $r(n)=1$. So in this case

$$
\lambda(n)=12(r(n)-1)+10\left(2^{s}-r(n)+1\right)-2=2\left(2^{s+2}+\left(2^{s}+r(n)\right)-2\right)
$$

for $r(n)>1$ and

$$
\lambda(n)=10 \cdot 2^{s}-2=2\left(2^{s+2}+2^{s}-1\right)
$$

for $r(n)=1$.

Thus the formula for the permutation complexity of the Thue-Morse word is

$$
\lambda(n)=\sum_{|u|=n} f(u)-\sum_{b \in B(n-1)} g(b)=2^{k+2}+2 b-2-2=2\left(2^{k+1}+b-2\right)
$$

for $n=2^{k}+b \geq 6$, where $0<b \leq 2^{k}$. 


\section{Concluding remark}

In this section we describe marked $l$-uniform binary morphisms for which we can apply our approach and calculate the permutation complexity of their fixed points. We note that Lemma 1 and Lemma 2 are key for our approach. Actually, if a marked $l$-uniform morphism satisfies Lemma 1 and Lemma 2 , then we can calculate the permutation complexity of its fixed point. So to apply our method we need that the morphism satisfies Lemma 1 and Lemma 2 Let us prove that for all marked $l$-uniform morphisms Lemma 1 holds only if it belong to the class $Q_{l}$.

Let $\varphi$ be a marked $l$-uniform morphisms. Let us prove that if $\varphi \notin Q_{l}$, then Lemma1 does not hold for $\varphi$. Recall that the partition of $\omega$ into blocks which are the images of its symbols is called correct.

Suppose that the first property in the definition of $Q_{l}$ does not hold for $\varphi$. There are two cases. In the first case $\varphi(0)=0 u 0 x$ for some word $x$ and $0 u 1$ is a subword of $\varphi(0)$ or $\varphi(1)$. Let $0 u 1$ be a subword of $\varphi(a)$, where $a \in\{0,1\}$. Then $\varphi(a)=z 0 u 1 y$ for some word $z$ and $y$. It is clear that the correct partition of $\omega$ contains at least one block $\varphi(a)$. Let $\omega_{j}$ lie in block $\varphi(a)$ in the correct partition of $\omega$ and $j \equiv|z|+1(\bmod l)$. Then $R_{\omega}(j)=0,0 u 1 y \ldots .$. Moreover we have $j \not \equiv 1(\bmod l)$. It is clear that the correct partition of $\omega$ contains at least one block $\varphi(0)$. Let $\omega_{i}$ lie in block $\varphi(0)$ in the correct partition of $\omega$ and $i \equiv 1(\bmod l)$. Then $R_{\omega}(i)=0,0 u 0 \ldots$ Then

$$
\gamma\left(R_{\omega}(i), R_{\omega}(j)\right)=\gamma(0,0 u 0 \ldots, 0,0 u 1 \ldots)=<
$$

and Lemma 1 does not hold for morphism $\varphi$.

In the second case $\varphi(0)=0 u 0 x$ for some word $x$ and $0 u$ is a suffix of $\varphi(0)$ or $\varphi(1)$. Let $0 u$ be a suffix of $\varphi(b)$, where $b \in\{0,1\}$. Then $\varphi(b)=z 0 u$ for some word $z$. Let $\omega_{j}$ lie in block $\varphi(b)$ which is followed by $\varphi(c)$ for some $c \in\{0,1\}$ in the correct partition of $\omega$ and $j \equiv|z|+1(\bmod l)$. Then $R_{\omega}(j)=0,0 u c \ldots$. Moreover we have $j \not \equiv 1(\bmod l)$. Let $\omega_{i}$ lie in block $\varphi(0)$ in the correct partition of $\omega$ and $i \equiv 1(\bmod l)$. Then $R_{\omega}(i)=0,0 u 0 \ldots$ Then

$$
\gamma\left(R_{\omega}(i), R_{\omega}(j)\right)=\gamma\left(R_{\omega}(i+|u|+1), R_{\omega}(j+|u|+1)\right) .
$$

If $c=1$, then $\gamma\left(R_{\omega}(i+|u|+1), R_{\omega}(j+|u|+1)\right)=<$ and Lemma 1 does not hold for morphism $\varphi$. If $c=0$, then inequalities $R_{\omega}(i+|u|+1)<R_{\omega}(j+|u|+1)$ and $R_{\omega}(i)>R_{\omega}(j)$ do not hold simultaneously. So Lemma 1 does not hold for $R_{\omega}(i)$ and $R_{\omega}(j)$ or $R_{\omega}(i+|u|+1)$ and $R_{\omega}(j+|u|+1)$. The case when the second property in the definition of $Q_{l}$ does not hold for $\varphi$ is similar.

Thus in a sense class $Q_{l}$ is optimal in the class of marked $l$-uniform morphisms for our approach.

\section{Acknowledgements}

I am grateful to A. E. Frid and S. V. Avgustinovich for helpful and stimulating discussions. I am also grateful to anonymous referees for valuable comments.

\section{References}

S. V. Avgustinovich. The number of distinct subwords of fixed length in the morse-hedlund sequence. Sibirsk. zhurnal issledovaniya operatsii, 1(2):3-7, 1994.

S. V. Avgustinovich, A. Frid, T. Kamae, and P. Salimov. Infinite permutations of lowest maximal pattern complexity. Theoretical Computer Science, 412:2911-2921, 2011. 
S. Brlek. Enumeration of factors in the thue-morse word. Discrete Appl. Math, 24:83-96, 1989.

J. Cassaigne. An algorithm to test if a given circular hd01-language avoids a pattern. In IFIP World Computer Congress'94, North-Holland, 1994. Elsevier.

J. Cassaigne. Complexité et facteurs spéciaux. Bull. Belg. Math. Soc., 4:67-88, 1997.

J. Cassaigne and F. Nicolas. Factor complexity in combinatorics. In V. Berthé and M. Rigo, editors, Combinatorics, Automata and Number Theory. University Press, Cambridge, 2010.

D. Fon-Der-Flaass and A. Frid. On periodicity and low complexity of infinite permutations. European $J$. Combin., 28(8):2106-2114, 2007.

A. E. Frid. On the subword complexity of infinite words generated by morphisms. Diskr. analiz $i$ issl. operacii, 4(1):53-59, 1997.

A. E. Frid. On uniform d01 words. STACS'98, Lect. Notes Comp. Sci., 1373:544-554, 1998.

A. E. Frid. On Combinatorial Properties of fixed points of morphisms. PhD thesis, Novosibirsk, 2000.

A. Luca and S. Varricchio. Some combinatorial properties of the thue-morse sequence and a problem in semigroups. Theoret. Comput. Sci., 63:333-348, 1989.

M. A. Makarov. On permutations generated by infinite binary words. Sib. Elektron. Mat. Izv., 3:304-311, 2006.

M. A. Makarov. On the permutations generated by the sturmian words. Sib. Math. J., 50(4):674-680, 2009.

A. Valyuzhenich. Permutation complexity of the fixed points of some uniform binary morphisms. In Proceedings of WORDS 2011, volume 63 of EPTCS, pages 257-264, 2011.

S. Widmer. Permutation complexity of the thue-morse word. Adv. in Appl. Math., 47(2):309-329, 2011. 